Forces on a circular cylinder at critical Reynolds

\title{
Unsteady forces on a circular cylinder at critical Reynolds numbers
}

\author{
O. Lehmkuhl, ${ }^{1,2, a)}$ I. Rodríguez, ${ }^{2}$ R. Borrell, ${ }^{2}$ J. Chiva, ${ }^{2}$ and A. Oliva ${ }^{2}$ \\ ${ }^{1)}$ Centre Tecnològic de Transferència de Calor (CTTC) Universitat Politècnica de \\ Catalunya (UPC), Spain \\ 2) Termo Fluids S.L. Avda. Jacquard, 977 1-E, 08222 Terrassa(Barcelona), \\ Spain
}

(Dated: 3 November 2014)

It is well known that the flow past a circular cylinder at critical Reynolds number combines flow separation, turbulence transition, reattachment of the flow and further turbulent separation of the boundary layer. The transition to turbulence in the boundary layer causes the delaying of the separation point and, an important reduction of the drag force on the cylinder surface known as the Drag Crisis. In the present work, large-eddy simulations of the flow past a cylinder at Reynolds numbers in the range $2.5 \times 10^{5}-6.5 \times 10^{5}$ are performed. It is shown how the pressure distribution changes as the Reynolds number increases in a asymmetric manner, occurring first on one side of the cylinder and then on the other side to complete the drop in the drag up to 0.23 at $R e=6.5 \times 10^{5}$. These variations in the pressure profile are accompanied by the presence of a small recirculation bubble, observed as a small plateau in the pressure, and located around $\phi=105^{\circ}$ (measured from the stagnation point). This small recirculation bubble anticipated by the experimental measurements is here well captured by the present computations and its position and size measured at every Reynolds number. The changes in the wake configuration as the Reynolds number increases are also shown and their relation to the increase in the vortex shedding frequency is discussed. The power spectra for the velocity fluctuations are computed. The analysis of the resulting spectrum showed the footprint of Kelvin-Helmholtz instabilities in the whole range. It is found that the ratio of these instabilities frequency to the primary vortex shedding frequency matches quite well the scaling proposed by Prasad and Williamson $\left(f_{K H} / f_{v s} \propto R e^{0.67}\right)$.

\footnotetext{
a)Electronic mail: cttc@cttc.upc.edu
} 
Forces on a circular cylinder at critical Reynolds

\section{INTRODUCTION}

Turbulent flow around bluff bodies such as a circular cylinder is of great interest for understanding fundamental fluid mechanics problems. This flow involves extremely complex physical phenomena such as flow separation due to the adverse pressure gradient, transition to turbulence and the shedding of vortices due to the interaction between both separated shear-layers ${ }^{1}$. According to Roshko ${ }^{2}$, depending on the characteristics of the flow it can be classified into different regimes: subcritical, critical, super-critical and trans-critical. In the subcritical regime $\left(R e=U_{\text {ref }} D / \nu \approx 10^{3}-2 \times 10^{5}\right)$, transition to turbulence occurs in the separated shear-layers with the drag coefficient remaining almost constant throughout the whole range and equal to $C_{D} \approx 1.2^{2-7}$. In this regime, the frequency of vortex shedding is also constant $S t=f_{v s} D / U_{\text {ref }} \approx 0.21$ whereas the vortex formation length decreases with the Reynolds number ${ }^{8}$.

In the range $2 \times 10^{5}<R e<5 \times 10^{5}$, also known as the critical regime ${ }^{9-11}$, there is a sharp decrease of the drag coefficient magnitude down to a minimum value of $C_{D} \approx 0.2$. In this regime, transition to turbulence first occurs in one of the boundary layers and it is characterized by the separation with further reattachment of the boundary layer, forming a bubble similar to that observed in the flow past airfoils at low-to-moderate Reynolds numbers ${ }^{12,13}$. This laminar separation bubble (LSB) on one side of the cylinder surface is the cause of asymmetric forces acting on the cylinder surface with the mean lift coefficient greater than zero $\left(C_{L}>0\right)$. Flow separation in the transitional shear-layers occurs further downstream at about $147^{\circ}$ (measured from the front stagnation point) ${ }^{14}$.

In the super-critical regime $\left(R e=5 \times 10^{5}-2 \times 10^{6}\right)$, characterized by the presence of two LSB on both sides of the cylinder surface, there is a plateau in the value of the drag coefficient $\left(C_{D} \approx 0.2\right)$ with symmetric separation at $\phi=148^{\circ 14-16}$. The wake is thinner than in the subcritical regime, with width lower than the cylinder diameter. A point of controversy is the existence of vortex shedding at these Reynolds numbers. In fact, there is a considerable scattering in the vortex shedding frequency measurements ${ }^{5,10,11,15}$, whereas other authors claim that there is not vortex shedding whatsoever ${ }^{2,16,17}$. Delany and Sorensen ${ }^{15}$ used a pressure transducer probe downstream the cylinder to measure vortex shedding. Their results show some scattering with values falling between $0.35-0.45$. On the other hand, Roshko ${ }^{2}$ did not observe vortex shedding at $R e<3.5 \times 10^{6}$. He attributed 
Forces on a circular cylinder at critical Reynolds

the inconsistency of his measurements with those of Delany and Sorensen to the narrow wake width and the fact that his probe was located at $[x, y] \equiv[7 D, 0.7 D]$ downstream the cylinder. Later, the measurements of Bearman ${ }^{10}$, Achenbach and Heinecke ${ }^{5}$ and Schewe $^{11}$ showed vortex shedding frequencies around $S t \approx 0.4-0.5$. Regarding the experimental tests by Achenbach and Heinecke, they did notice the suppression of vortex shedding for low aspect ratio cylinders and attributed this behavior to some three-dimensional effects and the consequent formation of a three-dimensional wake. On the other hand, their experiments at larger cylinder aspect ratios showed a quasi-regular flow fluctuation in this regime, in agreement with the previous experiments of Bearman ${ }^{10}$. Further experiments on smooth and rough cylinders by Shih et al. ${ }^{16}$ found neither vortex shedding at $S t>4 \times 10^{5}$ nor an increase in the Strouhal number up to 0.4 as previously reported.

The trans-critical regime $\left(R e=2 \times 10^{6}-3.5 \times 10^{6}\right)$ comes with a narrow-band vortex shedding at $S t \approx 0.27$ and the drag coefficient increasing again up to a value of $C_{D} \approx$ $0.5-0.7^{2,18}$. Indeed, there is no consensus in the magnitude of the drag coefficient at these Reynolds numbers as few experiments have been conducted so far. With a further increase beyond $R e>3.5 \times 10^{6}$, transition to turbulence in the boundary layer moves forward the stagnation point. No evidence of the LSB has been found in this regime ${ }^{2}$, thus being the separation of the flow fully turbulent. As a consequence of the upstream separation, compared to the super-critical regime, the wake becomes wider with rather high drag coefficient and base pressure.

Regarding the critical regime, many experimental works have been conducted; however, as the flow at these high Reynolds is very sensitive to small turbulence fluctuations, surface roughness, end conditions, etc., the scattering in the measurements is rather high. One of the pioneer experiments carried out were conducted by Wieselsberger ${ }^{3}$, who performed a series of measurements of the drag coefficient from laminar to super-critical Reynolds numbers, thus encompassing the critical regime.

Fage $^{4}$ and Fage and Falkner ${ }^{19}$ accomplished systematic measurements for determining the drag of circular cylinders, but also the distribution of the pressure and the skin friction along the cylinder circumference. Their experiments aimed at examining the boundary layer and its transitions on the cylinder. They reported on the change in the pressure profile as the flow enters the critical regime and the existence of an inflexion point in the pressure curve which marks the zone where the boundary layer transitions to turbulence. 
Forces on a circular cylinder at critical Reynolds

It is now of general consensus that the LSB observed on both sides of the cylinder surface and registered as a plateau in the pressure distribution, located after the position where the pressure reaches a local minimum, is a characteristic of critical and super-critical regimes. The resulting pressure distribution was observed at $R e=4.54 \times 10^{5}$ and $5.96 \times 10^{5}$ by Bursnall and Loftin ${ }^{9}$. The authors also showed that by forcing an early transition (typical of a higher Reynolds number), such LSB disappeared as transition moved ahead the location at which the LSB would be occurred. The presence of this LSB was extensively discussed by Tani $^{12}$ in his review, pointing out that this phenomenon was similar to that observed in airfoils at incidence. In fact, although not discussed in their work, it was also present in the measurements done by Fage and Falkner ${ }^{19}$ for those Reynolds numbers where the flow was critical, and it could be observed in both pressure distribution and skin friction around the cylinder. Further evidence of the LSB at those Reynolds numbers has been reported in the works of Bearman ${ }^{10}$, Achenbach and Heinecke ${ }^{5}$ and Schewe ${ }^{11}$, among others.

Another interesting feature of the flow in the critical regime is the asymmetry in the pressure distribution due to the formation of a LSB only on one side of the cylinder. So far, it has only been reported experimentally. The formation of this LSB is associated with the changes in the flow and the beginning of the drag crisis. Indeed, Bearman ${ }^{10}$ in his experiments from $R e=10^{5}$ to $7.5 \times 10^{5}$ observed this phenomenon at $R e=3.4 \times 10^{5}$. This bubble, on one side of the cylinder, caused an average magnitude of the lift coefficient $C_{L}$ greater than zero $\left(C_{L}>0\right)$. With the increase in the Reynolds number, a second bubble then appeared on the other side of the cylinder to complete the drop in the drag off to a value of 0.23 .

Later, Schewe ${ }^{11}$ studied this flow for a large range of Reynolds numbers from subcritical to trans-critical regimes, thus confirming the observations of Bearman and demonstrating that this is a fundamental characteristic of the flow and not a consequence of the test conditions. In fact, as previously observed by Bearman, he did also measured two discontinuous drops in the drag while increasing the Reynolds number.

Related to the change in the drag are the changes in the wake topology and the way separated shear-layers interact. Thus, variations in the vortex shedding frequency were also detected. In fact, the magnitude of the Strouhal number $\left(S t=f_{v s} D / U_{r e f}\right)$ was found to increase with the Reynolds number, with a discontinuous transition from 0.21 to 0.32 in the one-bubble zone to then rose up to 0.46 in the two-bubble zone ${ }^{10}$. Similar findings were also 
Forces on a circular cylinder at critical Reynolds

reported after Bearman ${ }^{10}$ by Achenbach and Heinecke ${ }^{5}$ and Schewe ${ }^{11}$, though with little difference in the values of the vortex shedding frequency.

Up-until-now, time accurate numerical simulations of the turbulent flow past a circular cylinder have been limited to low-to-moderate Reynolds numbers (see for instance Ma et al. ${ }^{20}$, Dong et al. ${ }^{21}$, Lehmkuhl et al. ${ }^{22}$ ). The main limitations of direct numerical simulations (DNS) techniques are due to the large range of instantaneous scales to be solved, which increase with the Reynolds number and require the use of very large computational resources. Hence, in order to get rid of such limitations, turbulent flow modeling such as Reynolds-averaged Navier-Stokes (RANS) models, detached eddy simulations (DES) and large-eddy simulations (LES) might be an alternative to tackle such complex phenomena at high Reynolds numbers. So far, due to limitations of the computational resources, numerical simulations of the flow in the critical regime are scarce. One of the first attempts was carried out by Celik and Shaffer ${ }^{23}$ using the standard $k-\epsilon$ RANS model in the range of Reynolds numbers of $10^{4}-10^{7}$. The results showed a fair agreement before separation, though after separation flow prediction failed due to the inability of the model used in capturing the flow dynamics in the wake. More recently, Vaz et al. ${ }^{24}$ compared different two- and threedimensional RANS and DES computations to experimental measurements at two Reynolds numbers of $R e=9 \times 10^{4}$ and $R e=5 \times 10^{5}$. They concluded that all models predicted forces far from the experimental values, especially at the higher Reynolds number. Travin et al. ${ }^{25}$ showed the capabilities of DES for dealing with this complex flow up to $R e=3 \times 10^{6}$. They obtained a reasonable agreement within experimental measurements, however they were not able of capturing the asymmetries in the flow even with the cylinder tripped on one side.

In addition to RANS and DES techniques, there have also been some numerical studies using LES at high Reynolds numbers. Unlike RANS, in LES only the smallest (sub-grid) scales of the flow are modeled, solving also all the temporal scales of the flow. This makes LES suitable for modeling the complex unsteady behavior of massive separated flows past bluff bodies ${ }^{26-28}$. Breuer ${ }^{29}$ performed challenging LES at the subcritical Reynolds number of $R e=1.4 \times 10^{5}$ and investigated the effect of two sub-grid scale (SGS) models. Although results were quite satisfactory, in special those obtained with the dynamic Smagorinsky model, further grid refinement did not improve the prediction of the flow. Later, Catalano et al. ${ }^{30}$ used LES with wall-modeling at super-critical Reynolds numbers of $5 \times 10^{5}, 10^{6}$ and $2 \times 10^{6}$. Results were very promising considering the grids used and were in fair agreement 
Forces on a circular cylinder at critical Reynolds

with state-of-the-art experimental results, especially for the two lower Reynolds numbers.

Sing and Mittal ${ }^{31}$ conducted two-dimensional DNS in a large range of Reynolds numbers up to $10^{6}$ aiming at studying the relation between shear-layer instabilities and the drag crisis, but their conclusions were limited due to the two-dimensional nature of the simulations. The flow over a rotating cylinder at $R e=1.4 \times 10^{5}$ and different spinning ratios from 0 to 2 was studied by means of LES by Karabelas ${ }^{32}$. Results for the stationary cylinder were in quite good agreement with the previous results by Breuer ${ }^{29}$. More recently, Moussaed et al. ${ }^{33}$ used a blending of a variational multi-scale LES (VMS-LES) with a RANS model for simulating the super-critical regime at $R e=6.5 \times 10^{5}-1.25 \times 10^{6}$. The pressure distribution along the cylinder circumference was reasonably good compared with experiments though they were not able of capturing the LSB in their simulations.

In spite of the large number of experimental studies conducted so far, in the critical regime there is a large scattering in the results obtained. This might be attributed mainly to the difficulties in accurate measuring this flow at these Reynolds numbers together with a large number of experimental issues such as wind tunnel blockage ratio, cylinder aspect ratio, turbulence intensity of the free-stream flow, cylinder end conditions and surface roughness, among others. Furthermore, most of these experimental studies have been concerned with measurement of drag forces, skin-friction and vortex shedding frequency. On the other hand, numerical studies have been focused on demonstrating the capabilities of numerical simulations to deal with such complex flow rather than provide more insight into the physics of the flow. Thus, considering the actual state-of-the-art this work aims at providing further insight into the fluid dynamic behavior of the flow past a circular cylinder at critical Reynolds numbers. To do this, LES of the flow at Reynolds numbers of $R e=2.5 \times 10^{5} ; 3.8 \times 10^{5} ; 5.3 \times$ $10^{5}$ and $6.5 \times 10^{5}$ are carried out. The analysis of different flow characteristic such as the formation of the small recirculation bubble on the cylinder surface, the delayed separation of the flow or the changes in the vortex shedding as the Reynolds number is increased are addressed. 
Forces on a circular cylinder at critical Reynolds

\section{MATHEMATICAL AND NUMERICAL MODEL}

\section{A. Governing equations}

The spatially filtered Navier-Stokes equations can be written as,

$$
\begin{aligned}
& \frac{\partial \bar{u}_{i}}{\partial x_{i}}=0 \\
& \frac{\partial \bar{u}_{i}}{\partial t}+\frac{\partial \bar{u}_{i} \bar{u}_{j}}{\partial x_{j}}-\nu \frac{\partial^{2} \bar{u}_{i}}{\partial x_{j} \partial x_{j}}+\rho^{-1} \frac{\partial \bar{p}}{\partial x_{i}}=-\frac{\partial \mathcal{T}_{i j}}{\partial x_{j}}
\end{aligned}
$$

where $\bar{u}$ and $\bar{p}$ stand for the filtered velocity and pressure, respectively. $\nu$ is the kinematic viscosity and $\rho$ the density of the fluid. In equation $2, \mathcal{T}_{i j}$ is the subgrid scale (SGS) stress tensor which has to be modeled. Its deviatoric part is given by,

$$
\mathcal{T}_{i j}-\frac{1}{3} \mathcal{T}_{k k} \delta_{i j}=-2 \nu_{s g s} \overline{\mathcal{S}}_{i j}
$$

where $\overline{\mathcal{S}}_{i j}$ is the large-scale rate-of-strain tensor, $\overline{\mathcal{S}}_{i j}=\frac{1}{2}\left(g_{i j}+g_{j i}\right)$ being $g_{i j}=\partial \bar{u}_{i} / \partial x_{j}$. $\delta_{i j}$ is the Kronecker delta. To close the formulation, an appropriate expression for the subgrid-scale viscosity should be provided. In this paper, the wall-adapting local-eddy viscosity model (WALE) ${ }^{34}$ is used. This model, proposed by Nicoud and Ducros ${ }^{34}$ evaluates the eddy viscosity using the square of the velocity gradient tensor. In its formulation, the SGS viscosity accounts for the effects of both the strain and the rotation rates of the smallest resolved turbulent fluctuations. In addition, it has a proper near-wall behavior $\left(\nu_{s g s} \propto y^{3}\right)$. The WALE model evaluates the eddy viscosity as,

$$
\nu_{s g s}=\left(\mathcal{C}_{w} \Delta\right)^{2} \frac{\left(\overline{\mathcal{V}_{i j}}: \overline{\mathcal{V}_{i j}}\right)^{\frac{3}{2}}}{\left.\left(\overline{\mathcal{S}_{i j}}: \overline{\mathcal{S}_{i j}}\right)^{\frac{5}{2}}+\overline{\left(\mathcal{V}_{i j}\right.}: \overline{\mathcal{V}_{i j}}\right)^{\frac{5}{4}}}
$$

in the above expression, $\overline{\mathcal{V}_{i j}}$ is the deviatoric part of the square of the velocity gradient tensor $\overline{\mathcal{V}_{i j}}=\frac{1}{2}\left(g_{i j}^{2}+g_{j i}^{2}\right)-\frac{1}{3} \delta_{i j} g_{k k}^{2}$ with $g_{i j}^{2}=g_{i k} g_{k j}$ and $\mathcal{C}_{w}$ is the model constant. Here a value of $\mathcal{C}_{w}=0.325$ is used.

\section{B. Numerical method}

The methodology for solving the governing equations has been previously used with accurate results for solving the flow over bluff bodies with massive separation by means 
Forces on a circular cylinder at critical Reynolds

of both DNS DN,35,36 and LES techniques $^{28,37}$. In this methodology, the governing equations have been discretized on a collocated unstructured grid arrangement by means of secondorder spectra-consistent schemes $^{38}$. Such schemes are conservative, i.e. they preserve the symmetry properties of the continuous differential operators and, ensure both stability and conservation of the kinetic-energy balance even at high Reynolds numbers and with coarse grids. Furthermore, as stated by Verstappen and Veldman ${ }^{38}$, these schemes constitute a good starting point for the formulation of SGS models. For the temporal discretization of the momentum equation (2) an explicit two-step second-order self-adaptive scheme on a fractional-step method has been used for the convective and diffusive terms ${ }^{39}$, while for the pressure gradient term an implicit first-order scheme has been implemented. Further details about the discretization can be found in Jofre et al. ${ }^{40}$ and Trias et al. ${ }^{41}$.

The resulting Poisson equation is then solved by using a direct Schur-Fourier decomposition method. This method takes advantage of the discretization used for solving the threedimensional domain. As three-dimensional meshes are here constructed by a constant-step extrusion of a two-dimensional unstructured grid, the span-wise coupling of the discrete Poisson equation yields circulant sub-matrices where a Fast Fourier Transform (FFT) based algorithm can be used. Then, the initially coupled three-dimensional system of equations is de-coupled into a set of two-dimensional sub-systems which are solved by means of a direct Schur complement decomposition method (for more details the reader is referred to Borrell et al. $\left.^{42}\right)$.

For the computational meshes reported in this work, partitions up to 1024 CPUs depending on the size of the computational grids were considered. In terms of wall-clock time, the cost of each simulation depends on the number of time-steps required for completing the whole simulation and the cost per time-step. Since in the current computations a direct Poisson solver is used, the latter is constant for a given mesh and number of CPUs. For instance, for the largest case, the mesh size was of about 83.2 million control volumes for which 1024 CPUs were used. For this case, the cost per iteration was of 0.324s. Considering that about 1.8 million of iterations were required, the cost per degree of freedom was $1.79 \mathrm{~s}$ for all the simulation. All computations were carried out on Marenostrum III Supercomputer which is based on Intel SandyBridge processors working at $2.6 \mathrm{GHz}$ and coupled by means of an Infiniteband network. 
Forces on a circular cylinder at critical Reynolds

\section{Computational domain and boundary conditions}

The flow past a circular cylinder at critical Reynolds numbers of $R e=2.5 \times 10^{5}$, $3.8 \times 10^{5}, 5.3 \times 10^{5}$ and $6.5 \times 10^{5}$ is considered. Here, the Reynolds number $R e=$ $U_{\text {ref }} D / \nu$ is defined in terms of the cylinder diameter $D$ and the free-stream velocity

$U_{\text {ref }}$. The cases have been solved in a computational domain of dimensions $[x, y, z] \equiv$ $[-16 D, 16 D] ;[-10 D, 10 D] ;[0,0.5 \pi D]$ in the stream-, cross-stream and span-wise directions respectively, with a circular cylinder of diameter D at $(0,0,0)$. Considering the computational domain, the dimension of the transverse direction corresponds with a blockage ratio of $L_{y} / D=5 \%$, which is well within the values reported in experiments (e.g. Spitzer ${ }^{43} 2 \%$, Bursnall and Loftin ${ }^{9} 2.2 \%$, Delany and Sorensen ${ }^{15} 4.8-14 \%$, Bearman ${ }^{10} 8.3 \%$, Schewe $^{11}$ $10 \%$, Achenbach ${ }^{14} 16 \%$ ).

The boundary conditions at the inflow consist of a uniform velocity $(\mathrm{u}, \mathrm{v}, \mathrm{w})=(1,0,0)$, slip conditions in the top and bottom boundaries of the domain, while at the outlet a pressurebased condition is used. At the cylinder surface, no-slip conditions are prescribed. As for the span-wise direction, periodic boundary conditions are imposed.

\section{Grid resolution}

As commented before, the governing equations are discretized on an unstructured mesh generated by the constant-step extrusion in the span-wise direction of a two-dimensional unstructured grid. For constructing the meshes used in the present simulations, in the region behind the cylinder where the level of turbulence of the flow is higher more control volumes have been clustered, while the grids have been stretched out towards the outer regions of the domain. Furthermore, it is worth noting that the boundary layer at the cylinder surface is well resolved, i.e. no wall functions are used. Thus, the meshes are designed so as to keep the non-dimensional wall distance $y^{+}<2$. To do this, a prism layer is constructed around the cylinder surface. A detail of the computational grid in the vicinity of the cylinder surface is depicted in Figure 1.

With all these criteria in mind the final meshes for each Reynolds number here adopted are given in Table I. It should be pointed out that although different grids have been considered for each of the cases solved, for the sake of brevity results presented in this work 
Forces on a circular cylinder at critical Reynolds
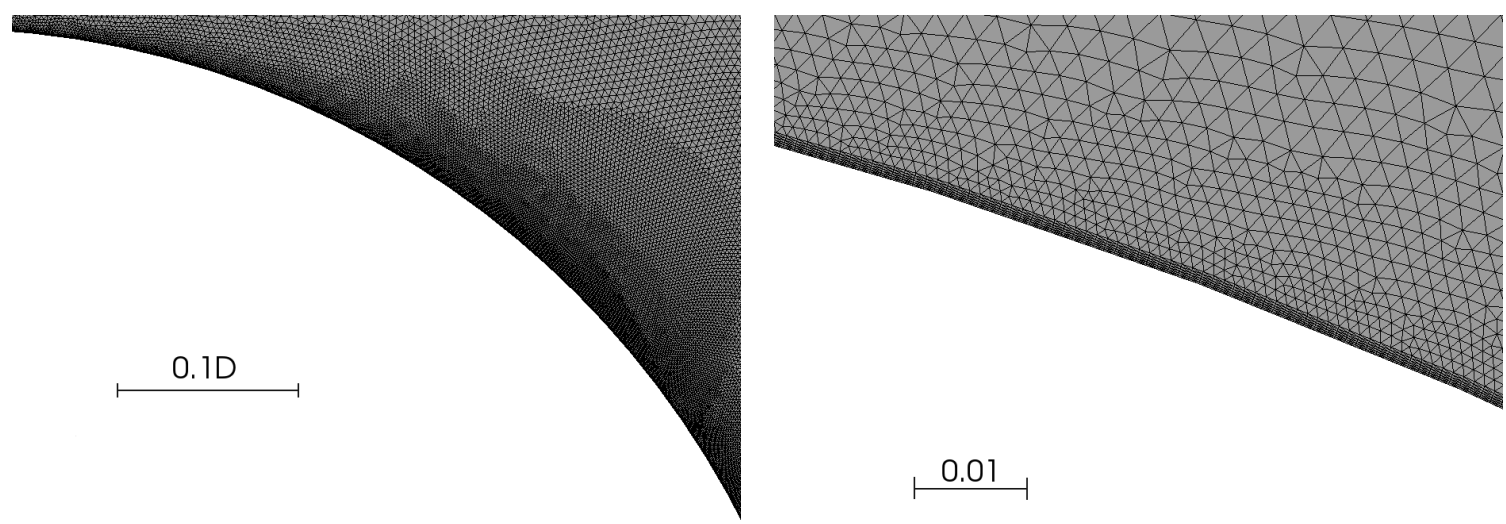

0.01

FIG. 1: Details of one of the computational meshes near the cylinder $(500516 \times 128 \approx 64$ million CVs).

TABLE I: Main parameters for the different computations. $N C V_{t}$ total number of control volumes; $N_{C V}$ plane number of control volumes in the plane; $N_{\text {planes }}$ number of planes in the span-wise direction

\begin{tabular}{cccc}
\hline \hline Re & $N C V_{t}\left[\times 10^{6}\right]$ & $N_{C V \text { plane }}$ & $N_{\text {planes }}$ \\
\hline $2.5 \times 10^{5}$ & 38.4 & 299683 & 128 \\
$3.8 \times 10^{5}$ & 48.6 & 379950 & 128 \\
$5.3 \times 10^{5}$ & 64.1 & 500516 & 128 \\
$6.5 \times 10^{5}$ & 83.2 & 650432 & 128 \\
\hline \hline
\end{tabular}

were obtained with the grids summarized in the table. In Appendix A some details about grid refinement studies conducted are presented. For the Reynolds numbers considered, transition to turbulence occurs just after the boundary layer detaches from the cylinder surface. Thus, in the present formulation the transition to turbulence is well captured by the model, i.e. no artificial mechanism for triggering this phenomenon to occur is imposed.

Regarding the span wise size of the domain, it should be borne in mind that this distance might be large enough to contain the largest scales, whereas at the same time grid resolution has to be sufficient to capture the relevant scales of the flow. Notice, that a compromise between accuracy and cost of the simulations has to be considered. Span wise two point 
Forces on a circular cylinder at critical Reynolds

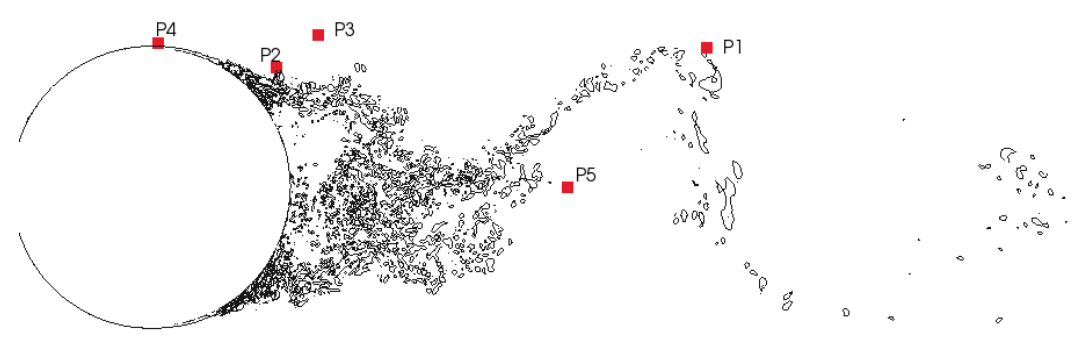

FIG. 2: Location of the numerical stations

correlations have been used to verify if the size of the domain is adequate. Two point correlations are defined as,

$$
\Re_{\phi \phi}(\mathbf{x}, \delta z)=\frac{<\phi^{\prime}(\mathbf{x}, t) \phi^{\prime}(\mathbf{x}+\delta z, t)>}{<\phi^{\prime 2}>}
$$

where $\mathbf{x} \equiv(x, y, z), \phi^{\prime} \equiv\left(u^{\prime}, v^{\prime}, w^{\prime}\right), \delta z$ is the spacing lag and $<\cdot>$ denotes averaging over time and space. In the present work different numerical probes have been located close to the cylinder and in the near wake (see figure 2). The time signals of these stations have then been recorded. The computed values of the two-point correlations for the stream wise $\left(R_{u u}\right)$, cross stream $\left(R_{v v}\right)$ and span wise velocity fluctuations $\left(R_{w w}\right)$ at probe $P 1 \equiv[x / D=2, y / D=0.5]$ for the different Reynolds numbers are given in figure 3. As can be seen, the correlations fall off to approximately zero at the half size of the domain. $R_{u u}$ and $R_{v v}$ are in general more narrower than $R_{w w}$ at every Reynolds number suggesting than the coherence in $w$ is larger than in the other two components. In particular, at $R e=2.5 \times 10^{5}$ it drops off more slowly and the correlation is not zero at the half span indicating the existence of a longer structure of size larger than $\pi / 4$. Although a larger domain would be desirable to accommodate all the structures and eliminate the effects of the periodic boundary condition, given the limited computational resources and the cost of the simulations, the span distance is a trade-off between enough resolution and sufficient distance in this direction. Thus, it was preferable to sacrifice the span size of the domain but keep a good resolution of the smallest scales.

\section{RESULTS}

For obtaining the numerical results presented, the simulations are started from an initially homogeneous flow field and then, are advanced in time until statistical stationary flow 
Forces on a circular cylinder at critical Reynolds

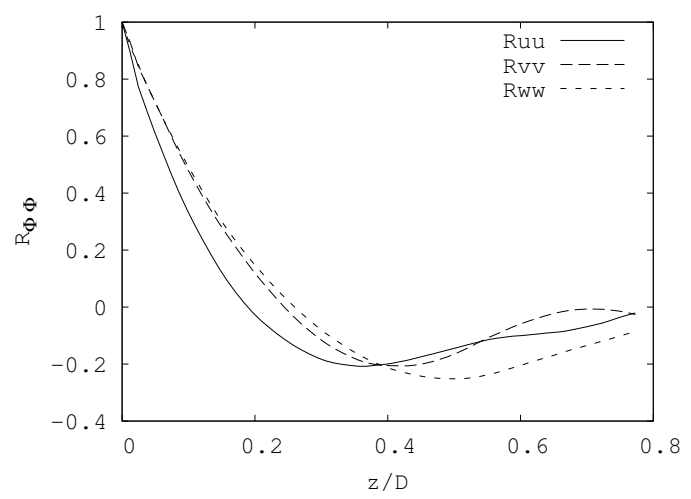

(a)

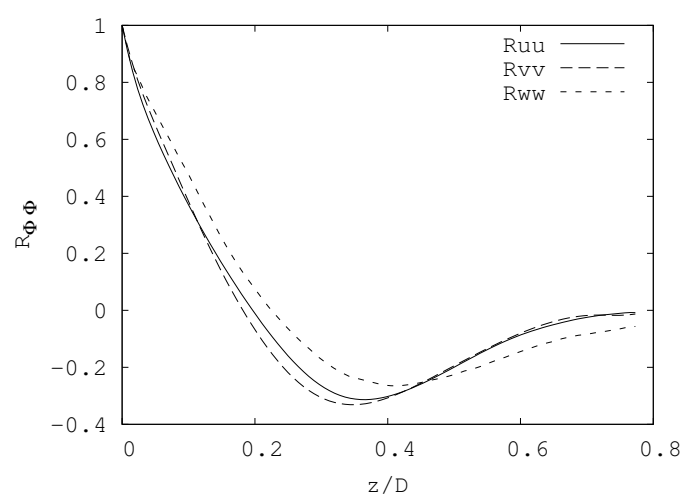

(c)

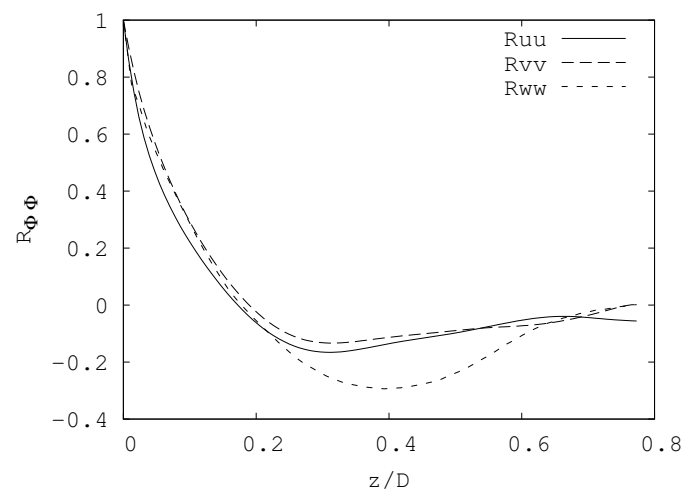

(b)

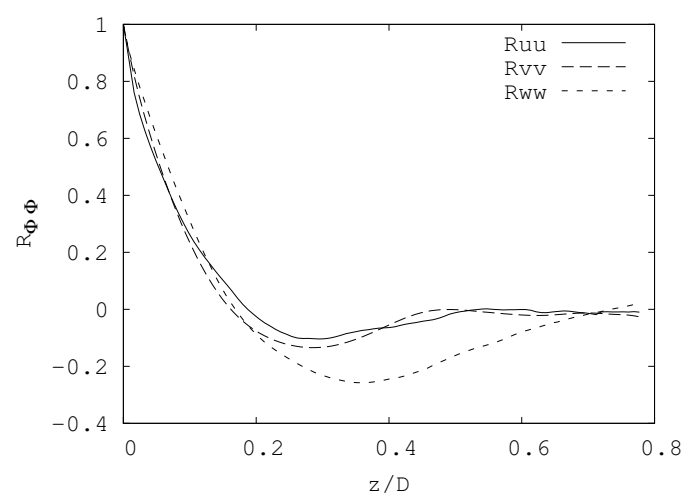

(d)

FIG. 3: Two-point correlations of the stream wise, cross stream and span wise velocity fluctuations in the near wake (a) at $R e=2.5 \times 10^{5}$; (b) at $R e=3.8 \times 10^{5}$; (c) at

$$
R e=5.3 \times 10^{5} ;(\mathrm{d}) \text { at } R e=6.5 \times 10^{5}
$$

conditions are achieved and the initial transient is completely washed out. Average statistics are then computed for a time span of about 25 shedding cycles, in order to assure that the flow is statistically converged.

\section{A. Local pressure and skin friction distributions}

The variation of the drag coefficient with the Reynolds number is plotted in Figure 4 together with the literature available measurements. As can be seen, at these Reynolds numbers, the measured data present a rather large scattering due to the difficulties associated with the experiments; i.e. sensitiveness to turbulence intensity, cylinder end conditions, 
Forces on a circular cylinder at critical Reynolds

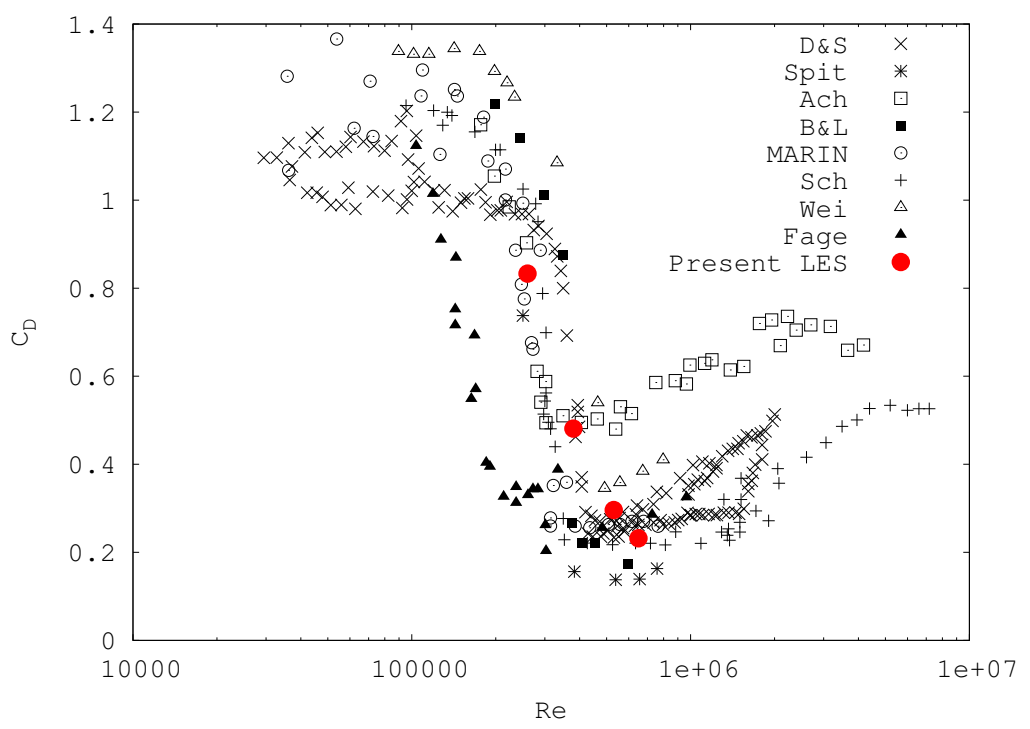

FIG. 4: Variation of the drag coefficient with the Reynolds number. Comparison with literature. (red solid circles) Present results; (crosses) Delany and Sorensen ${ }^{15}$; (stars) Spitzer $^{43}$; (squares) Achenbach ${ }^{5}$; (solid squares) Bursnall and Loftin $^{9}$ : (circles) MARIN ${ }^{24}$; (pluses) Schewe ${ }^{11}$; (triangles) Wieselsberger ${ }^{3}$; (solid triangle) Fage ${ }^{4}$

surface roughness, blockage ratio, among others. In spite of the large scattering in the reference data, results obtained with the present simulations show a fair agreement, being well within the range of dispersion of the experimental measurements. However, it should be pointed out that in some experiments such as those by Fage ${ }^{4}$, the onset of the critical regime occurs earlier at about $R e=10^{5}$, whereas compared to other experiments (e.g. Schewe ${ }^{11}$, Bursnall and $\operatorname{Loftin}^{9}$ ) it seems that in the present simulations the asymmetries in the flow are detected at lower Reynolds numbers. This suggests that the onset of the critical regime has here occurred slightly before than in those experiments. For instance, Bushnall and Loftin $^{9}$ observed the subcritical regime at $R e=2.45 \times 10^{5}$ and changes in the pressure distribution were measured for $R e>3.5 \times 10^{5}$, although no asymmetries were reported, the presence of a laminar separation bubble was detected at $R e=4.5 \times 10^{5}-5.96 \times 10^{5}$. Achenbach ${ }^{14}$ reported the onset of the critical regime at $R e=3 \times 10^{5}$, however, the drop in the drag in his measurements was rather high compared to other experiments (see figure 4). Schewe observed asymmetries in the flow at $R e=3.5 \times 10^{5}$, whereas Bearman ${ }^{10}$ reported the presence of a laminar separation bubble on one side of the cylinder at $R e=3.4 \times 10^{5}$ and the 
Forces on a circular cylinder at critical Reynolds

formation of a second bubble on the other side at $R e=3.8 \times 10^{5}$. In these experiments, the drop in the drag off to its minimum value followed a steep decrease (e.g Bearman between $R e=2 \times 10^{5}-4 \times 10^{5}$ and Schewe between $R e=2.5 \times 10^{5}-3.8 \times 10^{5}$ ), whereas in the present computations it occurs in a wider range, i.e. at $R e=2.5 \times 10^{5}$ the flow is in the critical regime and the end of this regime is measured when the drag reaches its minimum value at $R e=6.5 \times 10^{5}$. Some of these differences may stem from the fact that at these Reynolds numbers the changes in the drag occur very rapidly, being these changes comparable to the drag of the entire wind tunnel. This situation makes the system tunnel/cylinder unstable and thus, the drop in the drag of the cylinder results in a small increase in the wind tunnel speed $^{11,16}$. In these conditions it is difficult to keep constant the wind tunnel velocity, resulting in instabilities in the drag measurements.

The distribution of the local pressure on the cylinder surface at every Reynolds number is shown in Figure 5. Experimental measurements at comparable Reynolds numbers are also shown. The most noticeable feature of the profiles presented is the depression which occurs first on one-side (Figure 5a) and then on both sides (Figures 5b-5d) of the cylinder surface as the Reynolds number increases. Contrary to the subcritical regime where pressure distribution along the cylinder circumference remains almost unchanged regarding the Reynolds number, the variations observed in Figure 5 are a characteristic trait of the critical regime ${ }^{10,11}$. For the two lower Reynolds numbers, the pressure profiles are asymmetric, pointing out that boundary layer separation and transition to turbulence occurs at different locations in the top and bottom sides of the cylinder. As it will be also shown in section III B, transition to turbulence occurs earlier in one of the separated shear-layers, whereas on the other side the flow behavior is essentially as in the subcritical regime. The changes in the pressure profile account also for both the magnitude and the location at which minimum pressure occurs (see Table II). In the table, averaged data for the pressure minimum $\left(C_{p, \min }\right)$ and the angular position at which this minim occurs $\left(\phi_{C p, \min }\right)$, base pressure $\left(-C_{p}\right.$, base $)$, and drag $\left(C_{D}\right)$ and lift $\left(C_{L}\right)$ coefficients are summarized. Notice that both the minimum pressure and the location where it occurs are given for both sides of the cylinder surface.

At $R e=2.5 \times 10^{5}$, pressure minimum occurs near $70^{\circ}$ (measured following clock-wise direction from the stagnation point). The pressure distribution on this side of the cylinder is similar to that observed in subcritical flows and it compares quite well with that obtained by Cantwell and Coles $^{44}$ at the subcritical $R e=1.4 \times 10^{5}$ (see Figure $5 \mathrm{a}$ ). On the contrary, 
Forces on a circular cylinder at critical Reynolds

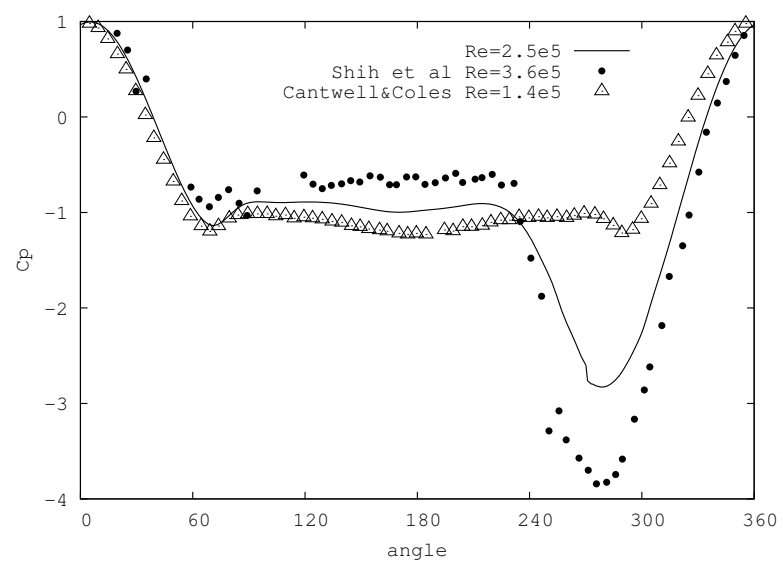

(a)

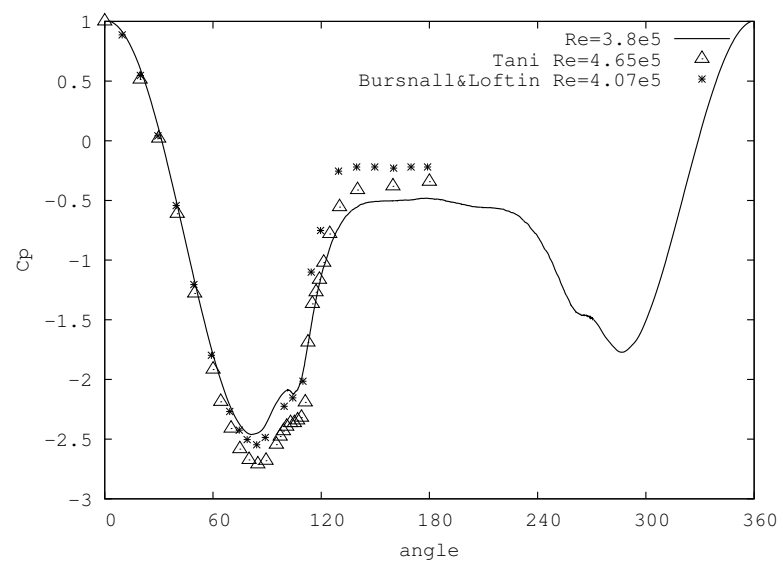

(b)

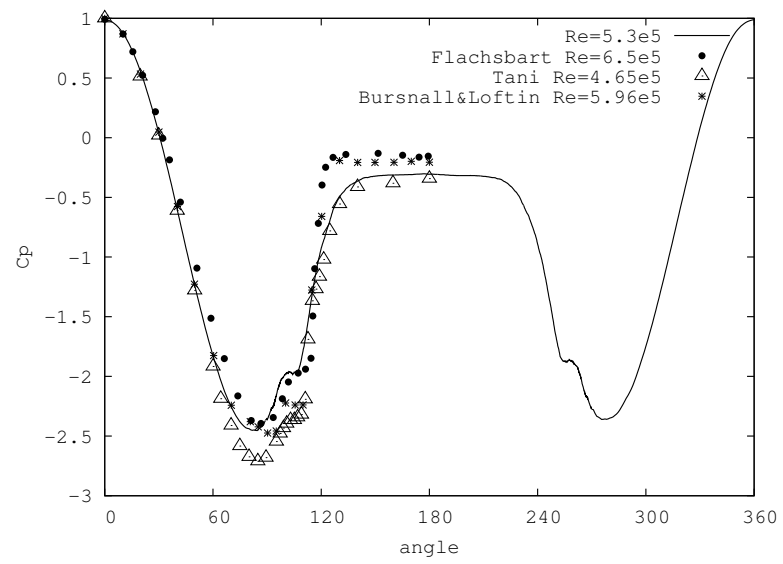

(c)

FIG. 5: For caption see facing page 
Forces on a circular cylinder at critical Reynolds

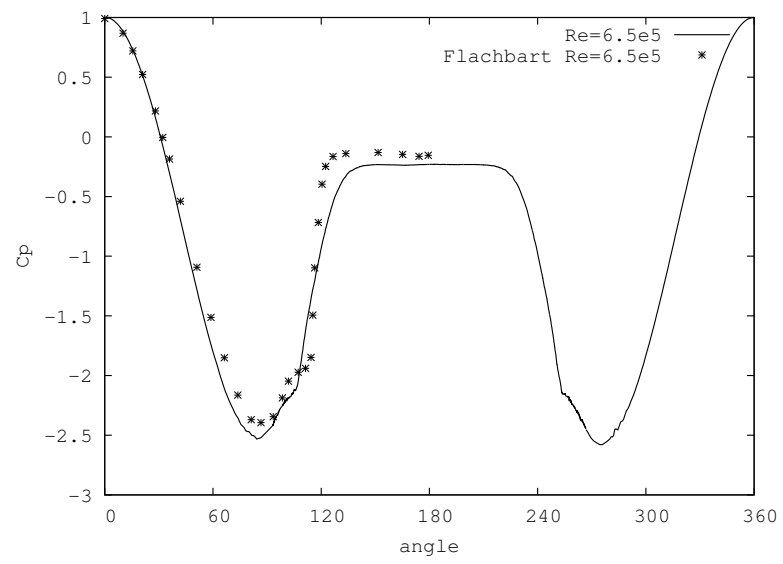

(d)

FIG. 5: Local pressure distribution at different Reynolds numbers. Comparison with the literature. (a) at $R e=2.5 \times 10^{5}$; (b) at $R e=3.8 \times 10^{5}$; (c) at $R e=5.3 \times 10^{5}$; (d) at

$$
R e=6.5 \times 10^{5}
$$

the pressure distribution on the other side of the cylinder presents a deep depression which reaches its minimum at about $82^{\circ}\left(278^{\circ}\right.$ ) (counter-clock wise direction from the stagnation point). These asymmetries were also reported in the experiments carried out by Shih et al. ${ }^{16}$

With the increase in the Reynolds number, the location of the minimum pressure moves towards the rear while it gets more negative (see also Table II). In fact, at $R e=6.5 \times 10^{5}$ pressure minimum approaches to $C_{p, \min }=-2.53$ at $85^{\circ}$. At the same time, the base pressure rises, thus increasing the magnitude of the pressure gradient. With all these variations in the pressure distribution along the cylinder circumference, the drop in the drag up to 0.23 in this range of Reynolds numbers is completed (see values of the drag coefficient in Table II).

Another remarkable trait is the plateau in the pressure observed at all critical Reynolds numbers (see Figure 5). This feature is registered around $\phi \approx 105^{\circ}$ (measured from the stagnation point) and just after the pressure reaches its local minimum. This plateau followed by a sudden pressure recovery is characteristic of the presence of a laminar separation bubble in this zone similar to that formed on pre-stalled airfoils at moderate Reynolds numbers ${ }^{9,12}$.

The combination of both the decrease in the pressure minimum and the increase in the back pressure makes larger the adverse pressure gradient, leading to the separation of the 
Forces on a circular cylinder at critical Reynolds

TABLE II: Pressure distribution main parameters. Pressure minimum $C_{p, \min }$ and its location $\phi_{C p, \min }$; cylinder base pressure $-C_{p, \text { base }}$ and drag $C_{D}$ and lift $C_{L}$ coefficients.

\begin{tabular}{cccccc}
\hline \hline Re & $C_{p, \min }$ & $\phi_{C p, \min }\left[^{\circ}\right]$ & $-C_{p, \text { base }}$ & $C_{D}$ & $C_{L}$ \\
\hline $2.5 \times 10^{5}$ & $-1.13 /-2.83$ & $70.5 / 278.6$ & 0.99 & 0.833 & -0.903 \\
$3.8 \times 10^{5}$ & $-2.46 /-1.77$ & $81.5 / 286.8$ & 0.48 & 0.481 & 0.245 \\
$5.3 \times 10^{5}$ & $-2.45 /-2.36$ & $82.8 / 276.7$ & 0.305 & 0.296 & 0.0614 \\
$6.5 \times 10^{5}$ & $-2.53 /-2.58$ & $85 / 275$ & 0.23 & 0.232 & 0.027 \\
\hline \hline
\end{tabular}

boundary layer. However, just after separation transition to turbulence takes place. Then, the shear-stresses which cause the transport of the momentum in the separated boundary layer are responsible for the closure of the LSB. This can be observed in Figure 6 where a close-up of both sides of the cylinder rear where the LSB appears is shown. In the figure, the averaged flow streamlines around this zone are depicted and the background is colored by the the averaged Reynolds stresses. As can be seen, the small recirculation bubble formed on the cylinder rear is well captured at all Reynolds numbers. At $R e=2.5 \times 10^{5}$, as expected, only one bubble on the bottom side is detected. This is in agreement with the local pressure profiles depicted in Figure 5a. Furthermore, the magnitude and location where shear-stresses are acting is quite different on both sides of the cylinder. While on the top side (subcritical side) transition to turbulence takes place off the cylinder surface, on the bottom side it comes closer to the cylinder being just in the location where the LSB is formed.

With the increase in the Reynolds number, the small recirculation bubble appears on both sides and, especially for $R e=5.3 \times 10^{5}$ and $R e=6.5 \times 10^{5}$, it is almost symmetric though a little bit larger at $R e=5.3 \times 10^{5}$. The size of the recirculation bubble at $R e=5.3 \times 10^{5}$ is around $14^{\circ}$ on average with a maximum height of about $0.0038 D$ at its center, whereas at $R e=6.5 \times 10^{5}$ it is around $8^{\circ}$ length and $0.003 D$ of height. The angular position where the LSB is located is also approximately given in the figure.

As commented before, the plateau observed in the pressure distribution is related to the presence of a LSB in the zone. This LSB also affects the skin friction on the cylinder surface which locally peaks at this region (see Figure 7 for details). In the figure, the local 
Forces on a circular cylinder at critical Reynolds
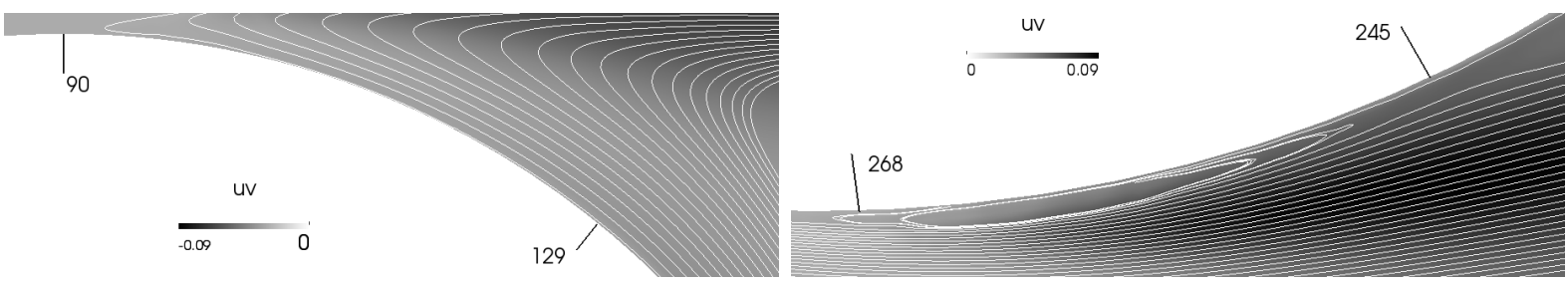

(a)
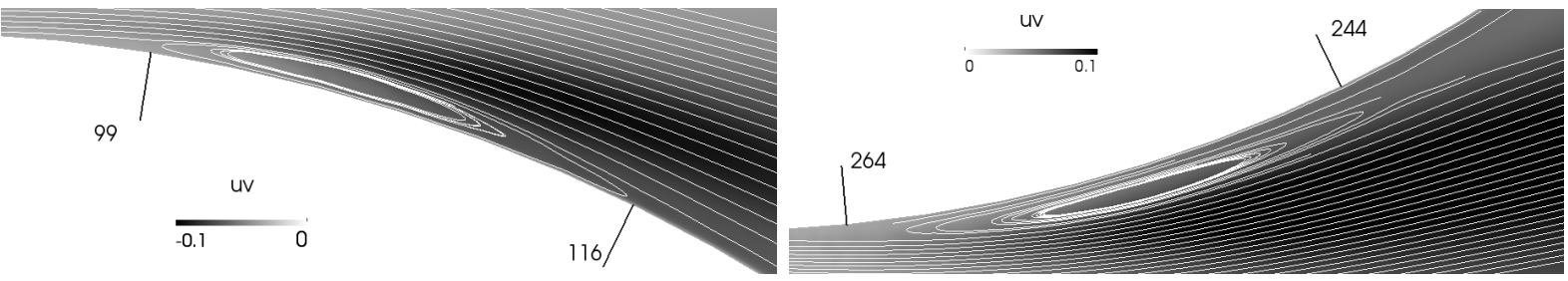

(b)
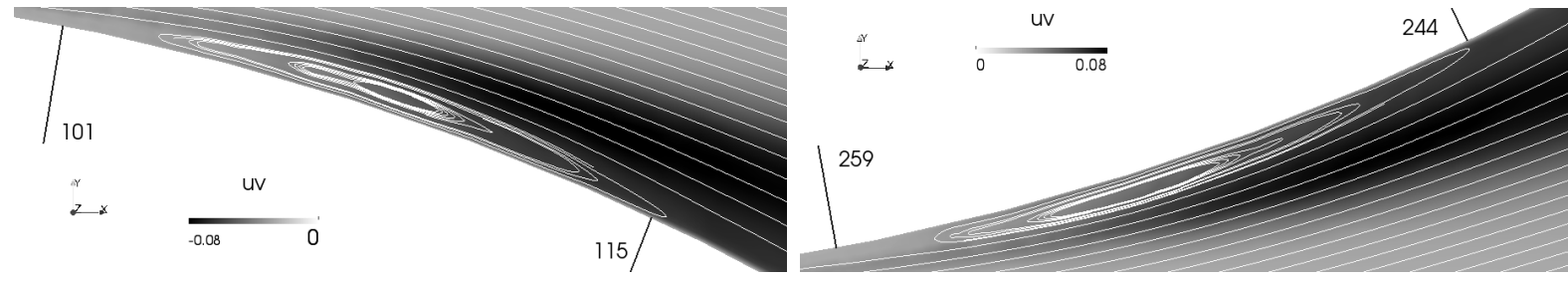

(c)
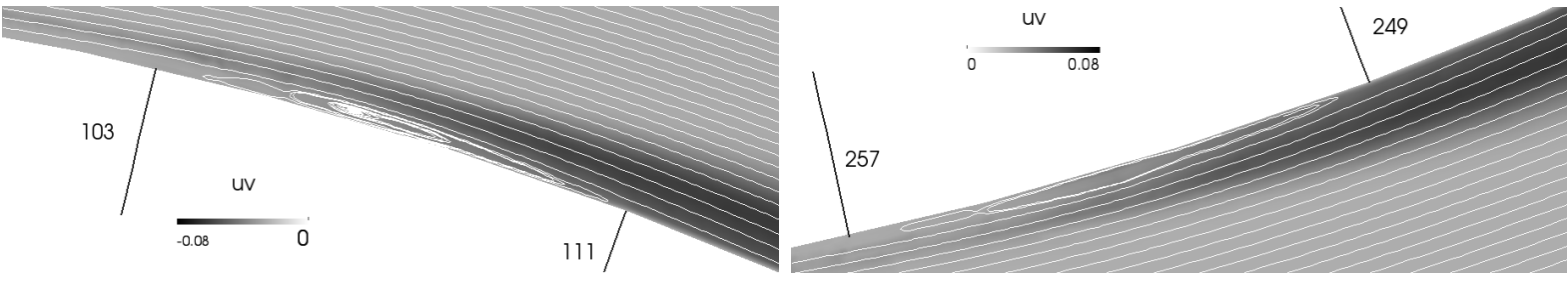

(d)

FIG. 6: Close-up of the LSB region. Flow average streamlines and shear-stresses at both sides of the cylinder surface (a) at $R e=2.5 \times 10^{5}$; (b) at $R e=3.8 \times 10^{5}$; (c) at

$$
R e=5.3 \times 10^{5} ;(\mathrm{d}) \text { at } R e=6.5 \times 10^{5}
$$

behavior of both variables is plotted. Indeed, the skin friction falls to a minimum followed by a steep increase in its magnitude, to then decrease again (see Figure 7). This increase in the skin friction magnitude evidences the existence of a turbulent boundary layer that further detaches at about $\phi_{s}=148^{\circ}$. The exact location where these inflection points occur for each Reynolds numbers are given in Table III. These changes were also pointed out by Fage and Falkner ${ }^{19}$, though in their experiments transition was triggered as earlier as at 
Forces on a circular cylinder at critical Reynolds

TABLE III: Angular positions of the recirculation bubble minimum $\phi_{\text {min }}$ and maximum $\phi_{\max }$ skin friction coefficient and flow separation $\phi_{s}$ on both sides of the cylinder surface.

\begin{tabular}{cccccccc}
\hline \hline \multirow{2}{*}{$R e$} & \multicolumn{3}{c}{ Top } & \multicolumn{4}{c}{ Bottom } \\
& $\phi_{\min }$ & $\phi_{\max }$ & $\phi_{s}$ & $\phi_{\min }$ & $\phi_{\max }$ & $\phi_{s}$ \\
\hline $2.5 \times 10^{5}$ & - & - & 91.5 & 270.6 & 252.9 & 217.7 \\
$3.8 \times 10^{5}$ & 99.5 & 106.95 & 145.2 & 266.8 & 262.8 & 218.8 \\
$5.3 \times 10^{5}$ & 100.1 & 110.1 & 148 & 259.4 & 250.7 & 212 \\
$6.5 \times 10^{5}$ & 102.2 & 107.9 & 148 & 257.9 & 252.6 & 212 \\
\hline \hline
\end{tabular}

$R e \approx 1.1 \times 10^{5}$. Later, Achenbach and Heinecke ${ }^{5}$ also observed this behavior of the skin friction. However, in their work the peak due to the transition from laminar to turbulent flow is located somewhat closer to the cylinder rear and the pressure distribution did not reflect the presence of a LSB, yet they measured the flow separation at $\phi_{s} \approx 147^{\circ}$ which is closer to that obtained in the present simulations.

\section{B. Instantaneous flow}

The instantaneous behavior of the flow at the different Reynolds numbers is depicted in Figure 8. Coherent structures are represented in the figure by means of the second invariant of the velocity gradient tensor $(Q \text {-criterion })^{45}$. The method identifies a vortical structure as a spatial region where rotation overcomes the strain. This invariant is defined as:

$$
Q=\frac{1}{2}\left(\Omega_{i j} \Omega_{i j}-\mathcal{S}_{i j} \mathcal{S}_{i j}\right)=-\frac{1}{2}\left(\frac{\partial u_{i}}{\partial x_{j}} \frac{\partial u_{j}}{\partial x_{i}}\right)
$$

where $\Omega_{i j}$ and $\mathcal{S}_{i j}$ stands for the skew-symmetric and symmetric components of $\nabla \mathbf{u}$.

In the figure, one can notice that regardless the Reynolds number and all the changes that occur in the critical regime, a von-Kármán vortex street is formed behind the cylinder. As it is discussed in the next section, vortex shedding is measured at all Reynolds numbers. In spite of the similarities, the wake topology suffers an important change with a dramatic reduction in the wake width and a variation in the location at which the boundary-layer separates, as it can also readily be seen from Figures 9 and 10 . The stream-wise distance 
Forces on a circular cylinder at critical Reynolds
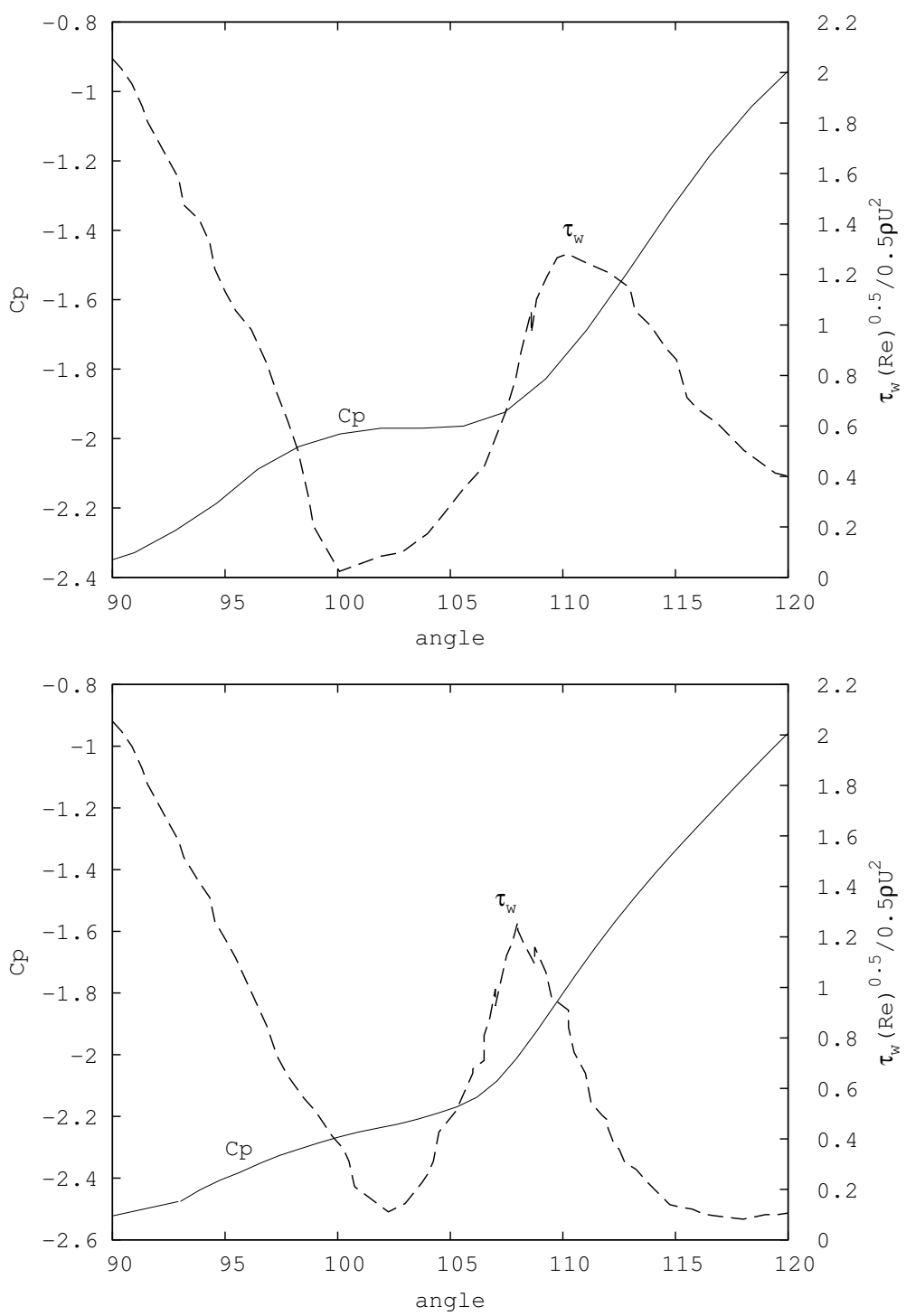

FIG. 7: Detail of the pressure distribution and the skin friction at the region where the LSB is detected. (a) $R e=5.3 \times 10^{5}$, (b) $R e=6.5 \times 10^{5}$

between the vortices shed also diminishes as a consequence of the increase in the vortex shedding frequency (see next section). This gives the wake a more compact aspect as can be observed at $R e=6.5 \times 10^{5}$, where several vortices are detected for the same length of the wake when compared to the lower Reynolds numbers (in the figure counter-clock wise (CCR) rotating and clock-wise (CR) rotating vortices are marked for a qualitative comparison).

At Reynolds number of $R e=2.5 \times 10^{5}$ (see Figure 8a), even when the flow is entering the critical regime, the broad wake resembles that formed behind a circular cylinder at 


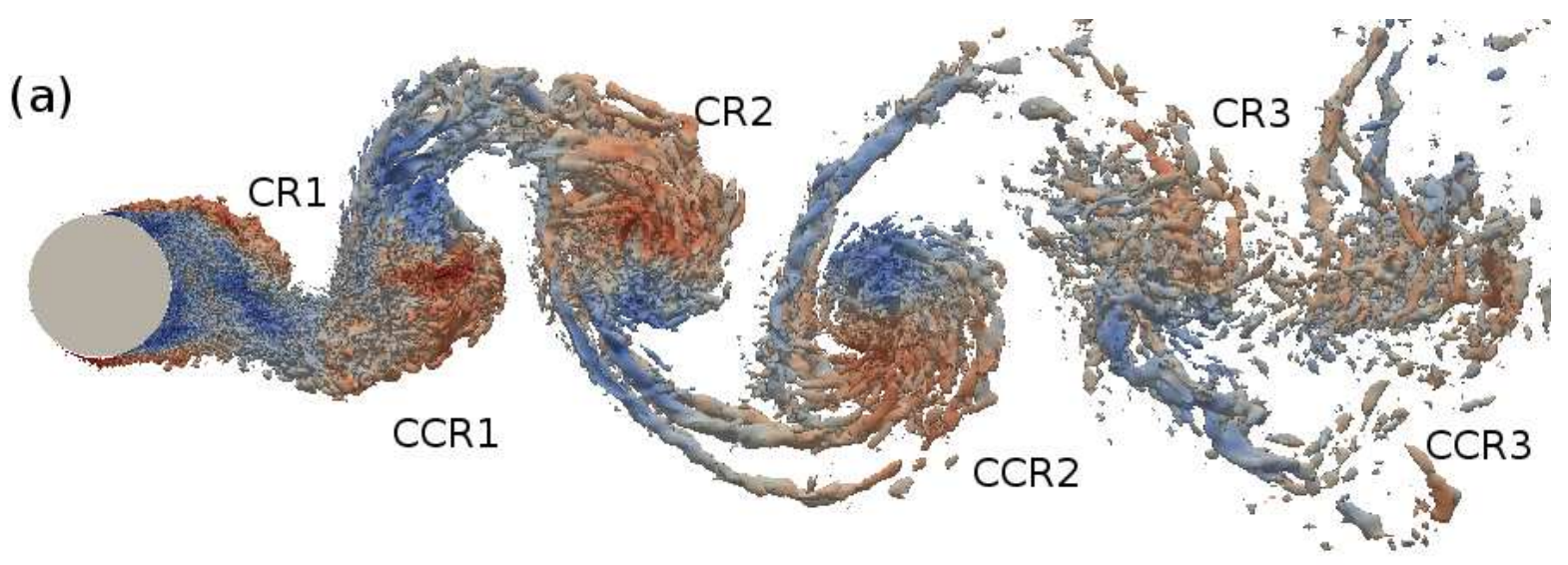

(b)
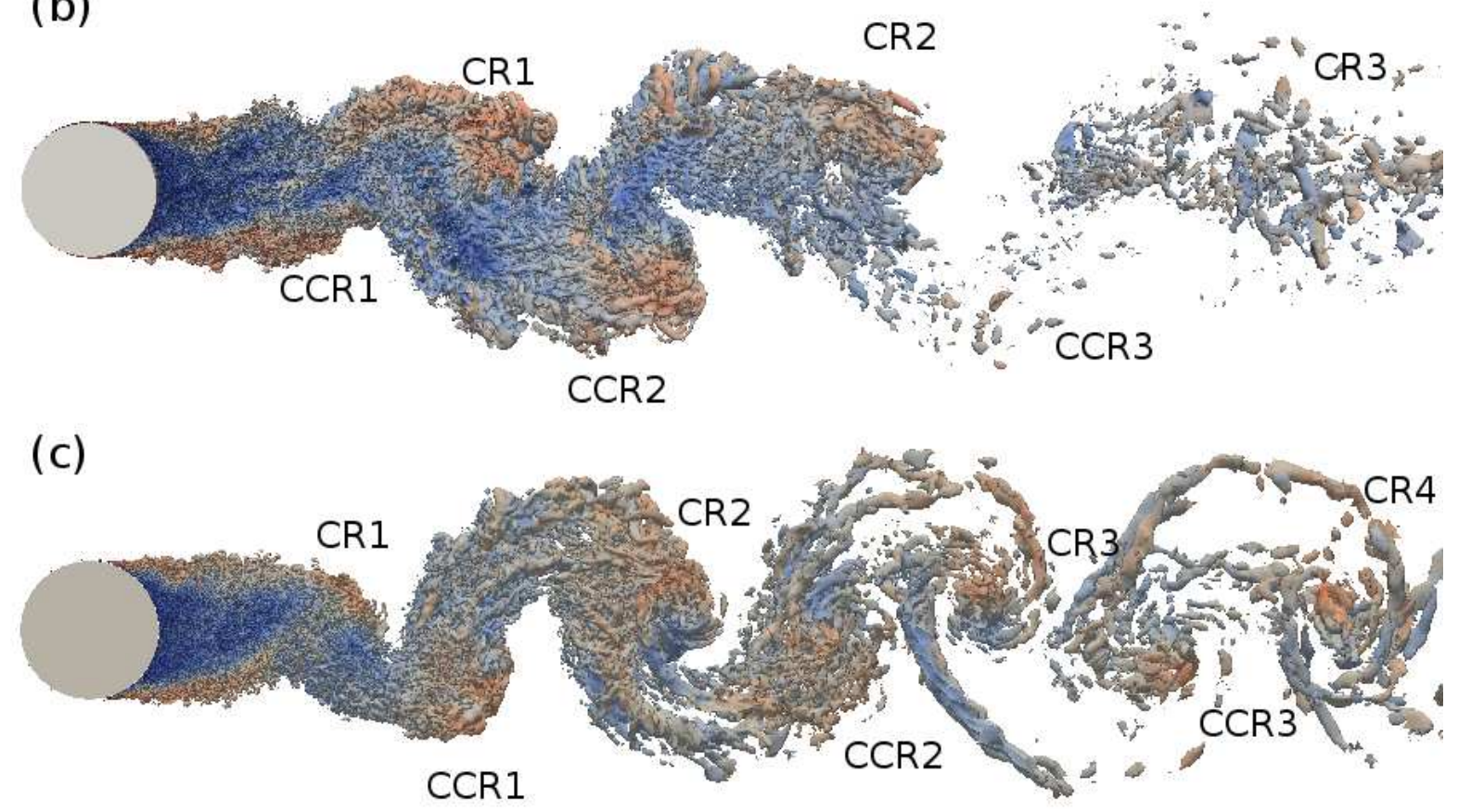

(d)

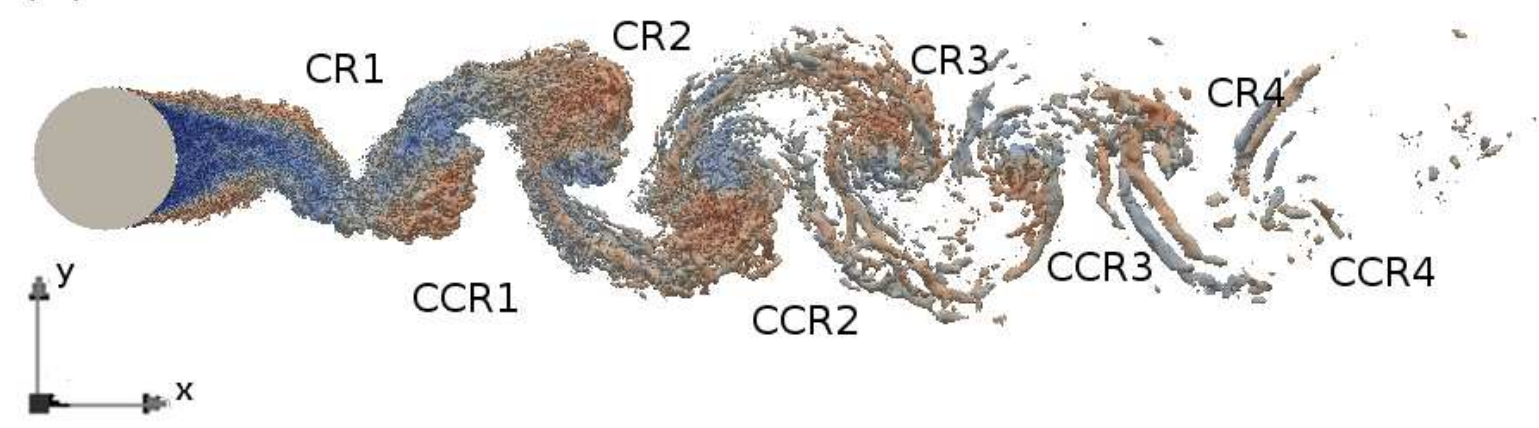

FIG. 8: Instantaneous wake configuration. Vortical structures represented by isocountours of $\mathcal{Q}=20$ colored by the velocity magnitude (a) $R e=2.5 \times 10^{5}$, (b) $R e=3.8 \times 10^{5}$, (c)

$$
R e=5.3 \times 10^{5}, \text { (d) } R e=6.5 \times 10^{5}
$$


Forces on a circular cylinder at critical Reynolds

subcritical Reynolds numbers, with almost parallel shear-layers detaching from the cylinder surface. These shear-layers roll-up downstream the separation point, forming alternating vortices which are shed into the wake as can be seen in the figure (see for instance ${ }^{46}$ ). The top boundary layer separates laminarly as it does in the subcritical regime ${ }^{6}$. In the figure, CR1 vortex is being formed as the top shear-layer wraps-up, while irrotational fluid entrainment occurs on the other side of the wake (see also Figures 9 and 10). Notwithstanding the flow separation is asymmetric, the vortex formation process is similar to that described by Gerrard $^{1}$. Furthermore, the presence of one-bubble on one-side of the cylinder surface, as it was shown in section III A by means of the pressure distribution, is well illustrated in Figure 9. In the figure, the instantaneous velocity magnitude in the near wake behind the cylinder is depicted. On the bottom side of the cylinder, it can be seen how flow separation has been delayed as a consequence of the increase of the pressure gradient (the difference between the minimum pressure and that at the cylinder rear). As the flow accelerates on this side of the cylinder, the process leading to the transition is triggered by the perturbations in the boundary layer (see Figures 10a and 11a). This makes the transition location in the detached boundary layer to come closer to the separation point which allows the flow reattachment and the formation of the LSB on this side of the cylinder. This is in well agreement with the average pressure profile obtained at this Reynolds number (see Figure 5a), where the pressure distribution on the top side is typical of subcritical flows whereas in the bottom side is critical.

At $R e=3.8 \times 10^{5}$, the flow is well within the critical regime. The pressure minimum has started to decrease on the other side of the cylinder (see Figure 5b), which together with the increase in the back pressure accelerates the flow triggering the transition just after separation, although separation is non-symmetric (see Figures 10b and 11b). The combined effects of flow separation, transition to turbulence and the increase in the shear-stresses, makes the flow to reattach causing the formation of asymmetric LSBs and delaying the final separation of the flow (see also figure $9 \mathrm{~b}$ ).

At $R e=5.3 \times 10^{5}$, the wake has not completed yet its critical transformation, however symmetry is almost attained and the wake width has been reduced (see Figure 9c). Flow instabilities close to the un-separated boundary layer are almost symmetric and can be observed at an angular position larger than $\phi=90^{\circ}$ (Figure 11c). The formed eddies (Figure 10c) delay the separation on both sides of the cylinder which finally occurs at 
Forces on a circular cylinder at critical Reynolds

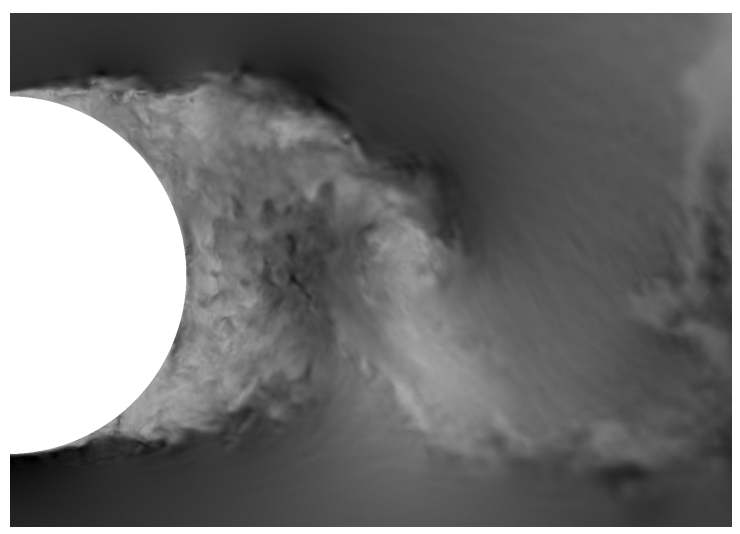

(a)

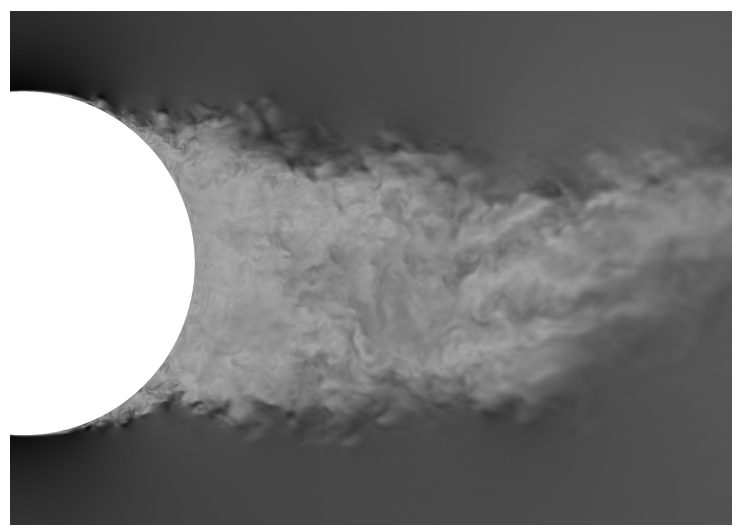

(c)

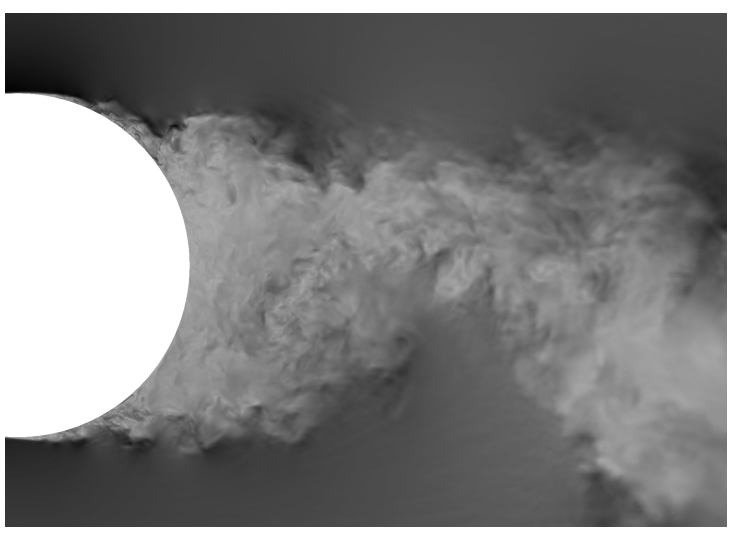

(b)

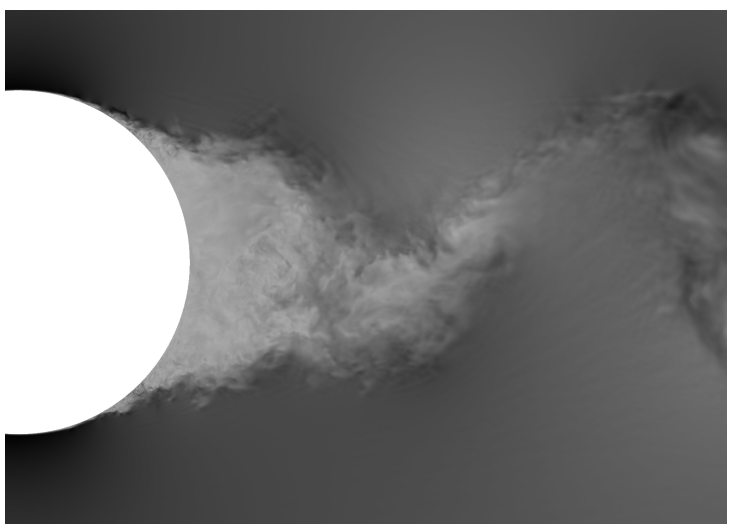

(d)

FIG. 9: Instantaneous velocity magnitude profiles in the near wake. (a) $R e=2.5 \times 10^{5}$; (b) $R e=3.8 \times 10^{5}$; (c) $R e=5.3 \times 10^{5}$; (d) $R e=6.5 \times 10^{5}$

around $\phi=148^{\circ}$ as qualitatively can be seen from these figures. Further downstream the separation point, the shear-layers interact with each other near the wake center line, but the shedding of vortices occurs at a higher frequency as will be discussed in the next section.

With the further increase in the adverse pressure gradient at $R e=6.5 \times 10^{5}$ topological changes are now accomplished (see Figures 8d, 9d and 10d). This increase in the pressure difference accelerates the flow and eventually makes the turbulent shear-layers to collapse in the wake center line. As this is happening, the recirculation zone behind the cylinder shrinks and the wake width gets smaller than that at $R e=5.3 \times 10^{5}$ (see also Figures $11 \mathrm{c}$ and $\mathrm{d}$ ) . 
Forces on a circular cylinder at critical Reynolds

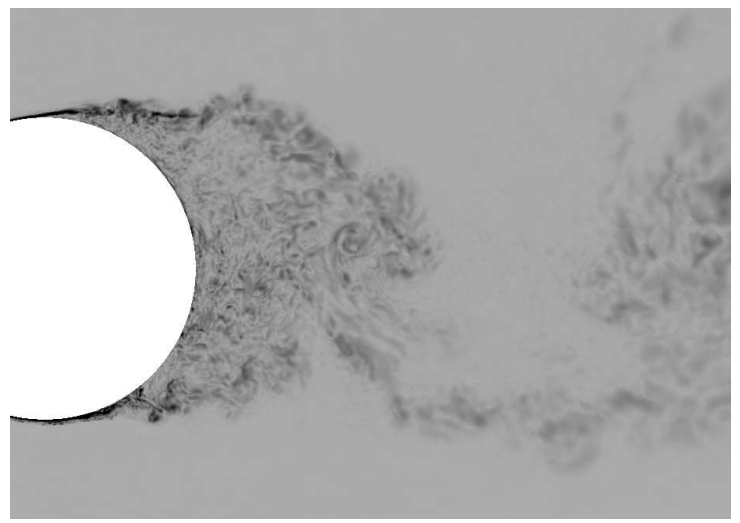

(a)

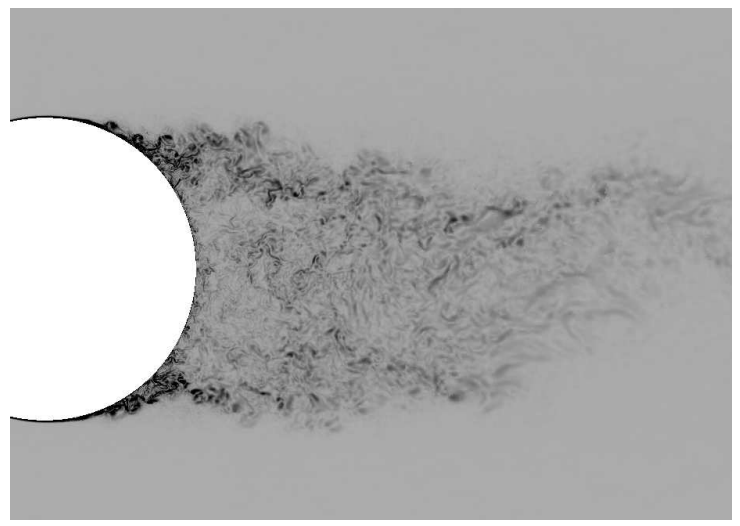

(c)

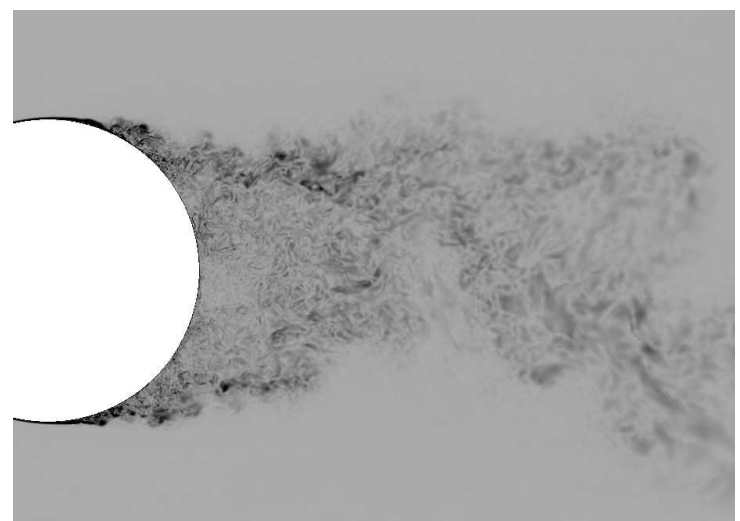

(b)

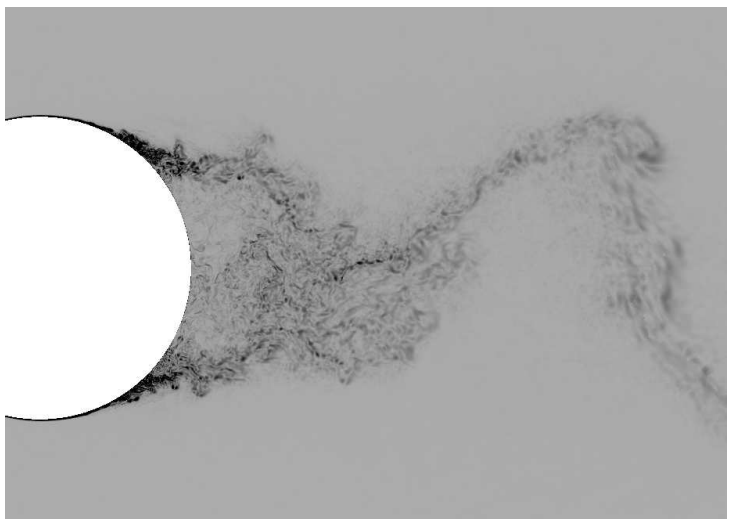

(d)

FIG. 10: Instantaneous vorticity profiles in the near wake. (a) $R e=2.5 \times 10^{5}$; (b)

$$
R e=3.8 \times 10^{5} ;(\mathrm{c}) R e=5.3 \times 10^{5} ; \text { (d) } R e=6.5 \times 10^{5}
$$

\section{Energy spectra}

Numerical probes have been located at different positions in the near wake, in order to capture the instantaneous behavior and spectrum of energy at the different Reynolds numbers studied. For the unevenly sampled velocity components time series data, energy spectrum is then calculated by using the Lomb periodogram technique ${ }^{47}$ and, by averaging the resulting spectrum in the span-wise direction. Figure 12 shows the resulting power spectrum of the cross-stream velocity fluctuations for the different Reynolds numbers. Although velocity components have been sampled at different stations, in the figure energy spectra are given for the near wake at $x / D=2$ and $y / D=0.5$. As can be seen, with the exception of the energy spectrum at $R e=3.8 \times 10^{5}$, a sharp peak at the vortex shedding frequency is 
Forces on a circular cylinder at critical Reynolds

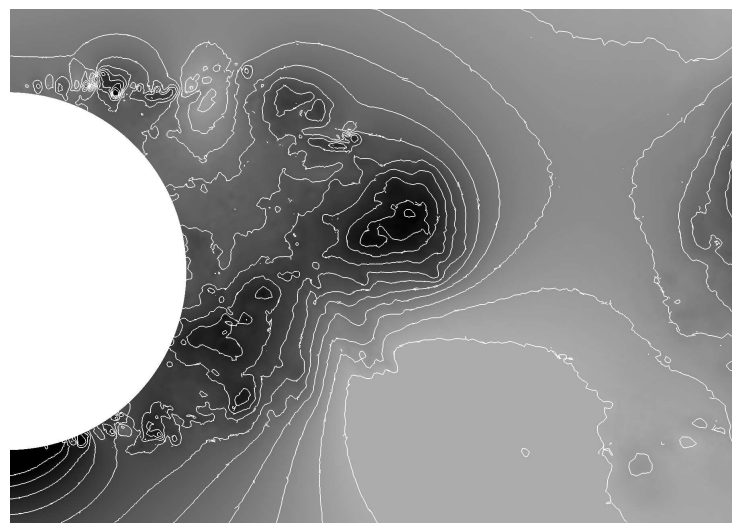

(a)

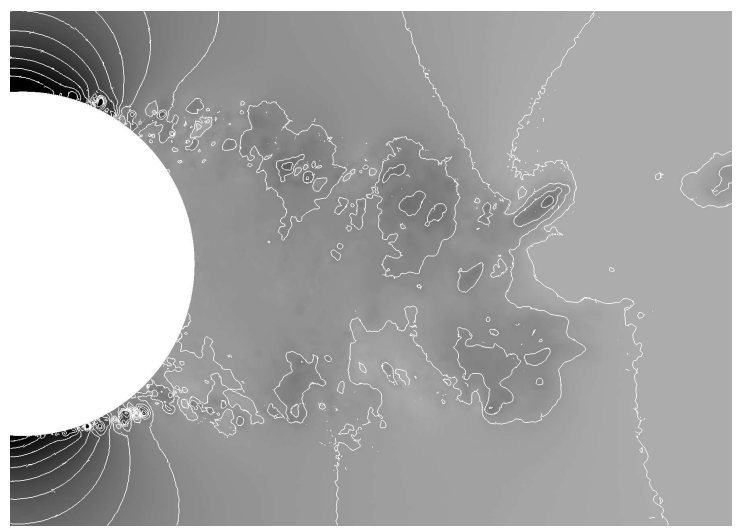

(c)

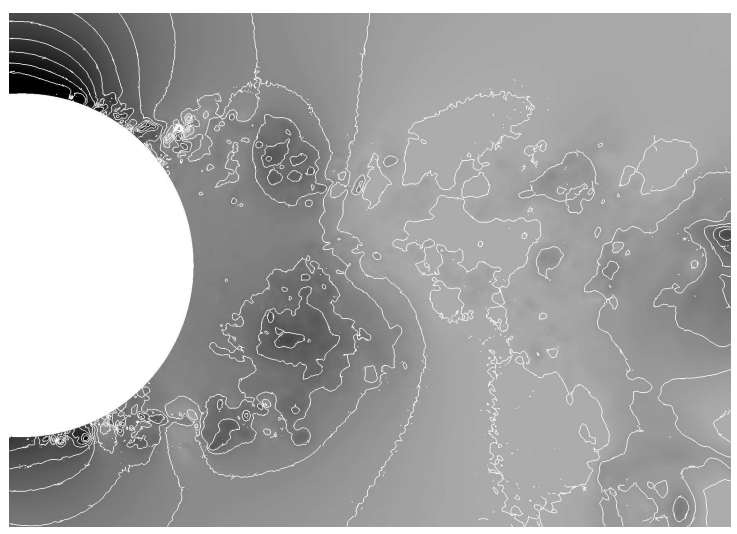

(b)

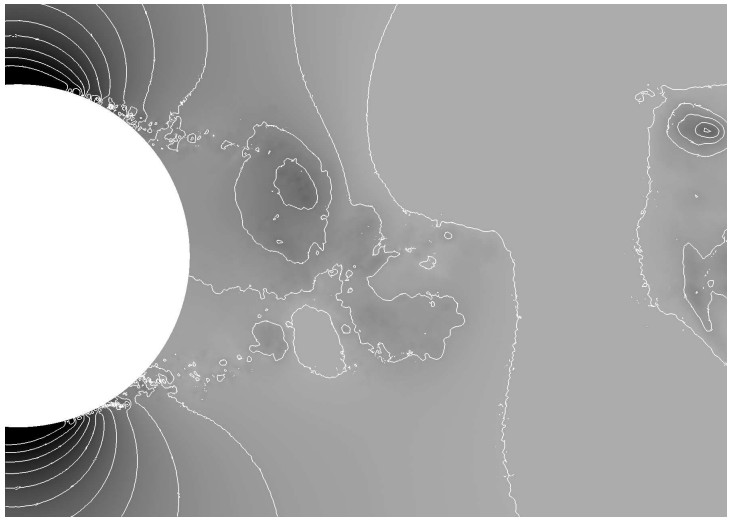

(d)

FIG. 11: Instantaneous pressure contours. (a) $R e=2.5 \times 10^{5}$; (b) $R e=3.8 \times 10^{5}$; (c)

$$
R e=5.3 \times 10^{5} ; \text { d) } R e=6.5 \times 10^{5}
$$

observed at all Reynolds numbers. However, the frequency at which energy peaks increases as the Reynolds number increases as can readily be seen in Table IV. For comparison, experimental results at comparable Reynolds numbers are also given.

These changes in the vortex shedding frequency have been reported before in the literature by Bearman ${ }^{10}$, Achenbach and Heinecke ${ }^{5}$ and Schewe $^{11}$. In the experiments of Bearman and Schewe, they both reported an increase in the vortex shedding frequency as the drag coefficient approaches to its minimum value. Bearman directly related these changes with the occurrence of one-bubble on one side of the cylinder surface and the second jump in the frequency with the establishment of the second bubble on the other side of the cylinder.

However, in the present computations, the discontinuous change from one-bubble to twobubble flow is not detected. Conversely, changes in the wake configuration and in the vortex 
Forces on a circular cylinder at critical Reynolds

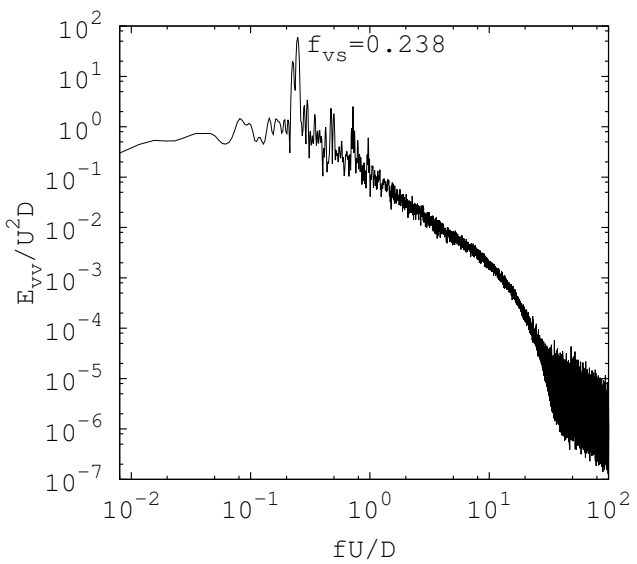

(a)

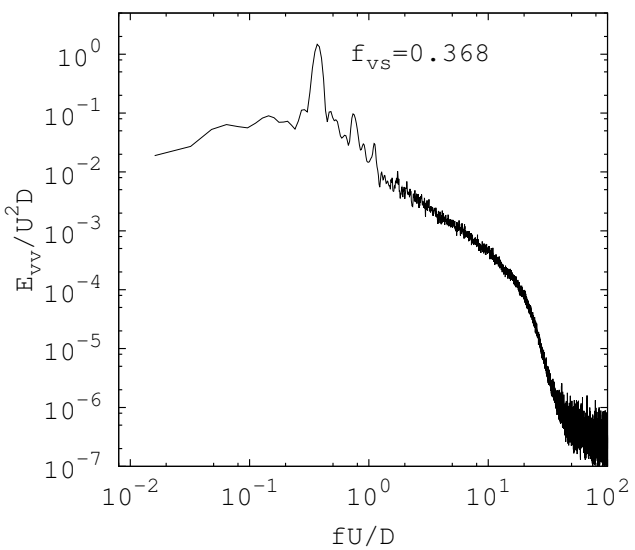

(c)

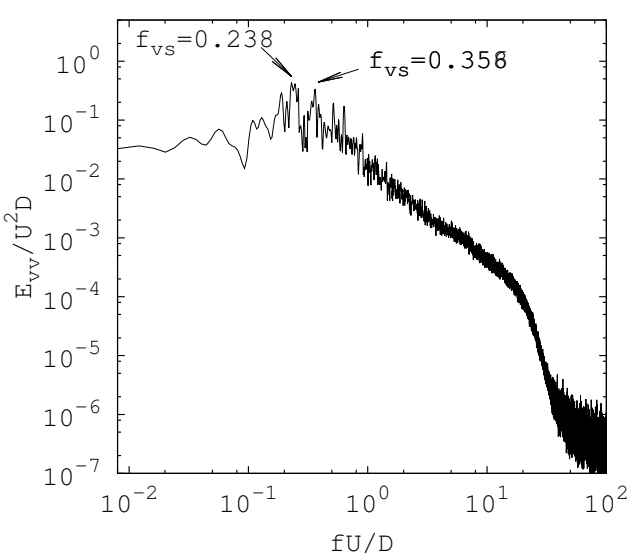

(b)

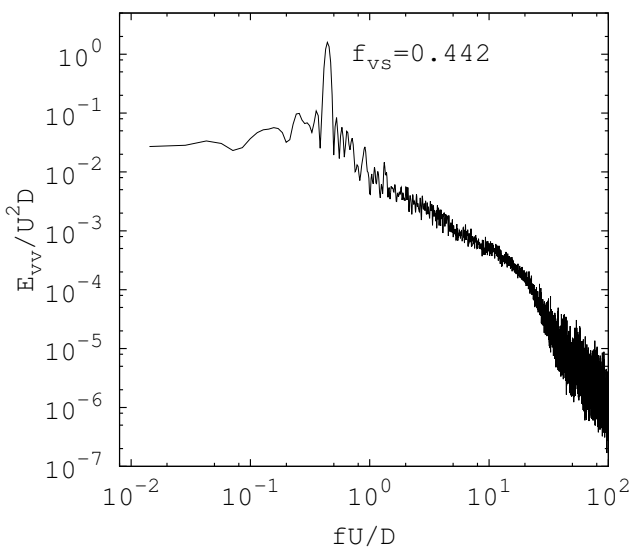

(d)

FIG. 12: Energy spectra of the cross-stream velocity fluctuations at $x / D=2, y / D=0.5$ at different Reynolds numbers. (a) $R e=2.5 \times 10^{5}$; (b) $R e=3.8 \times 10^{5}$; (c) $R e=5.3 \times 10^{5}$;

$$
R e=6.5 \times 10^{5}
$$

shedding regime occur more gradually. On one hand, at $R e=2.5 \times 10^{5}$ when the one-bubble flow regime is detected, vortex shedding frequency was $f_{v s}=0.238$, a value closer to that reported for subcritical flow $\left(f_{v s} \approx 0.2\right)$ rather than the value $f_{v s} \approx 0.35$. The last one should correspond with the one-bubble regime according to Bearman ${ }^{10}$ observations. On the other hand, at $R e=3.8 \times 10^{5}$ when the second bubble starts to form and, due to the asymmetry in the flow, the vortex shedding process loses coherence and two less energetic peaks at frequencies $f_{v s}=0.238$ and $f_{v s}=0.358$ are detected instead of a clear vortex shedding frequency peak (see Figure 12b). This loss of coherence might be attributed to the asymmetric vortex shedding as one of the shear-layers is bending towards the wake center line 
Forces on a circular cylinder at critical Reynolds

whereas the other remains more stream wise aligned. This makes the interaction between both shear-layers more random and gives the wake a less coherent appearance. Notice also that this double peak is also observed in the energy spectrum of the shear-layer (see figure 13). In fact, the frequencies detected correspond with the values measured at $R e=2.5 \times 10^{5}$ and at $R e=5.3 \times 10^{5}$ pointing out a fluctuation between both configurations as the second bubble is not yet completely formed. As a result, the wake configuration and the vortices formed are not as coherent as they are at $R e=2.5 \times 10^{5}$ or at the higher Reynolds numbers, as can also be seen from the vortical structures depicted in Figure 8b.

Furthermore, at $R e=5.3 \times 10^{5}$, when the second bubble is formed and the symmetry in the flow is almost re-established (see Figure 12c and Table IV), the frequency measured was $f_{v s} \approx 0.36$, and its value rose up to $f_{v s} \approx 0.44$ at $R e=6.5 \times 10^{5}$. These changes in the frequency of the vortex shedding seem to be related with the distance between both shear-layers, which is shrinking as the Reynolds number approaches to $R e=6.5 \times 10^{5}$, rather than with the presence of one-bubble flow as stated by Bearman ${ }^{10}$. This is somewhat in disagreement with the previous experiments ${ }^{10,11}$. However, it should be considered that in these experiments Reynolds number increments are carried out by small increments of the wind tunnel speed ${ }^{11}$. Under these circumstances, the measurements might be influenced by the previous state. Moreover, as it was also pointed out by Schewe ${ }^{11}$, fluctuations in the transition states (when the one-bubble flow was detected) were the largest and the wind tunnel speed was influenced by the transition states, as far as the drag changes in the cylinder were not small enough compared with the drag of the tunnel. Similar issues were also reported by Shih et al. ${ }^{16}$. One then wonders whether these instabilities when varying the Reynolds number, would influence the measurements of the vortex shedding frequency.

Conversely, numerical experiments carried out in the present work start from homogenous flow being each case independent with each other and cannot exhibit then, the hysteresis registered experimentally ${ }^{11}$. In these results, the minimum drag coefficient measured corresponds also with the wake configuration where shear-layers are brought closer together which is consistent with both the local pressure and the energy spectra measurements. Yet, topological changes do not affect the fundamentals of the vortex formation mechanism. When the shear-layers come closer due to the acceleration of the flow, their interaction is promoted and thus, the periodic vortex shedding takes place at a higher frequency. This statement is in agreement with the observations made by Gerrard $^{1}$ about the mechanism of vortex 
Forces on a circular cylinder at critical Reynolds

\begin{tabular}{lcccc}
\hline \hline$R e$ & $2.5 \times 10^{5}$ & $3.8 \times 10^{5}$ & $5.3 \times 10^{5}$ & $6.5 \times 10^{5}$ \\
\hline Present results & 0.238 & $0.238 / 0.358$ & 0.368 & 0.442 \\
Bearman $^{10}$ & 0.19 & 0.32 & 0.45 & 0.44 \\
Achenbach\&Heinecke $^{5}$ & 0.228 & $0.34\left(2.9 \times 10^{5}\right)$ & 0.51 & 0.51 \\
Schewe $^{11}$ & 0.2 & $0.31\left(3 \times 10^{5}\right)$ & 0.45 & 0.44 \\
\hline \hline
\end{tabular}

TABLE IV: Variation of the Strouhal number with the Reynolds number. Comparison with the literature results.

shedding in bluff bodies.

A fact that would require further attention is whether at $R e>4 \times 10^{5}$ vortex shedding ceases to occur as reported in some experiments (see for instance ${ }^{2,16,17}$ ). However, as has been shown, vortex shedding has been clearly measured at both $R e=5.3 \times 10^{5}$ and $R e=6.5 \times 10^{5}$. The reason why some investigators did not detect vortex shedding at $R e>4 \times 10^{5}$ is not clear. At these Reynolds numbers the flow is quite unstable and aspects such as a low cylinder aspect ratio or vibrations in the wind tunnel can trigger three-dimensional effects in the wake and the loss of coherence in the vortex shedding. Indeed, Achenbach and Heinecke ${ }^{5}$ observed these effects when they experimented with low aspect ratio cylinders and in such cases no regular signal was detected. These effects disappeared when larger aspect ratio cylinders were the used. It would be interesting to induce some three dimensional effects in order to clarify this issue. However, at this time this remains a subject for further research.

In addition to the vortex shedding frequency, the analysis of the energy spectrum can provide information about the instability of the shear-layers. It is well known that KelvinHelmholtz $(\mathrm{KH})$ instabilities in the shear-layer play a major role in the transition to turbulence. These instabilities lead to the formation of small-scale vortices which eventually grow up and feed the large-scale Kármán vortices. Since the work by Bloor ${ }^{8}$, consistent studies have been performed to visualize and measure the frequency of these instabilities ${ }^{48-50}$. The contribution of the $\mathrm{KH}$ instability occurs at different scales than that of the vortex shedding and its footprint can be identified by means of a Fourier analysis. However, the signature of $\mathrm{KH}$ instabilities is rapidly absorbed within the turbulent background fluctuations, and thus cannot be detected by all probes in the near wake. In order to detect KH instabilities 
Forces on a circular cylinder at critical Reynolds

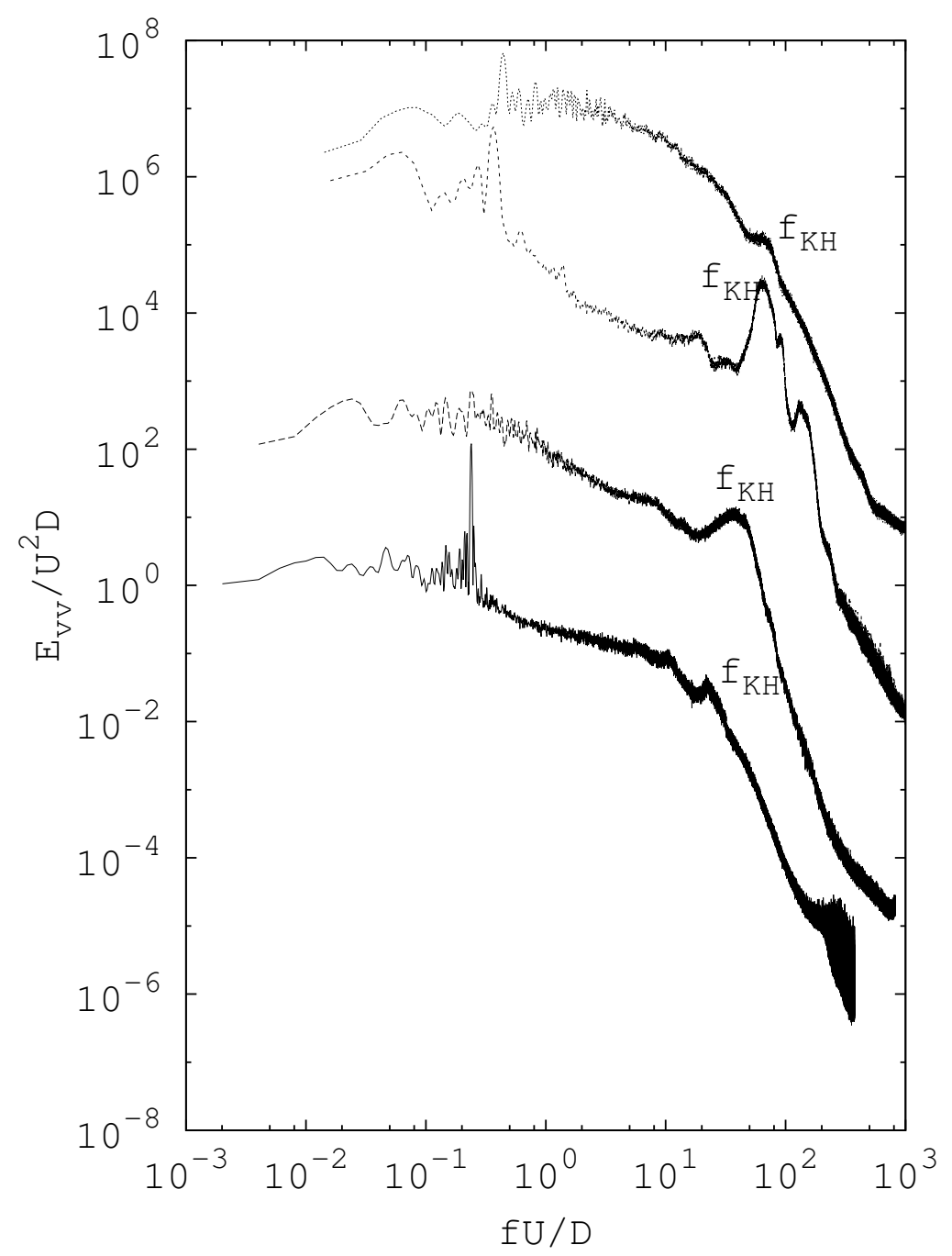

FIG. 13: Shear-layer instabilities frequency as a function of the Reynolds number. From bottom to top: $R e=2.5 \times 10^{5}, R e=3.8 \times 10^{5}, R e=5.3 \times 10^{5}$ and $R e=6.5 \times 10^{5}$. The $\mathrm{KH}$ frequency is marked in the spectra as $f_{K H}$

frequency, probes should be placed in the shear-layers or close to them. Then, the energy spectrum of selected probes (see figure 2 for details) has been computed (see figure 13).

At the Reynolds numbers studied, the onset of instabilities are located in the attached boundary layer (see also figure 11). Thus, KH instabilities were measured at probe P3 for $R e=2.5 \times 10^{5}, \mathrm{P} 4$ for $R e=3.8 \times 10^{5}$ and $R e=5.3 \times 10^{5}$ and $\mathrm{P} 5$ for $R e=6.5 \times 10^{5}$. In these locations, the footprint of these instabilities is captured as a broadband energetic peak at frequencies larger than that of the vortex shedding. The energy contained in the spectra of shear-layer instabilities at $R e=2.5 \times 10^{5}$ and $R e=6.5 \times 10^{5}$ is not that high, 
Forces on a circular cylinder at critical Reynolds

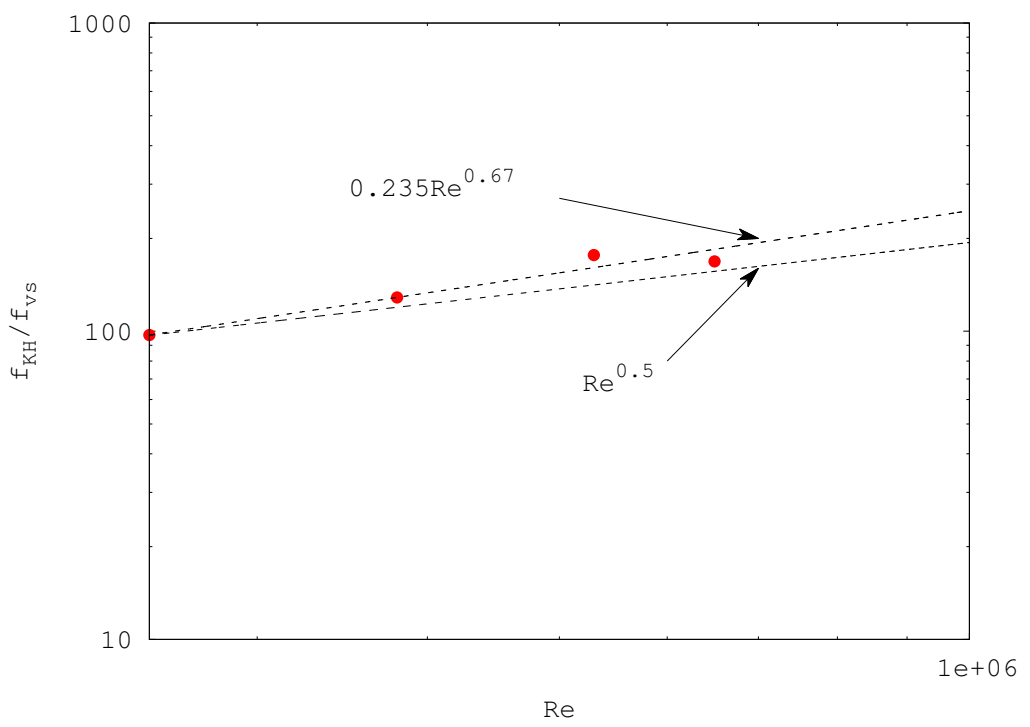

FIG. 14: Variation of ratio of the shear-layer frequency to the vortex shedding with Reynolds number. The correlation proposed by Prasad and Williamson ${ }^{51,52}$ and the $\propto R e^{0.5}$ scaling are also plotted

as the probes depicted are located a tad off the separated shear-layer. Yet, the broadband signature can be well captured. On the other hand, the energy spectra at $R e=3.8 \times 10^{5}$ and $R e=5.3 \times 10^{5}$, which correspond with probes located at the cylinder shoulder show more energetic peaks, as these probes are located well within the onset of these instabilities. These observations suggest that, similar to the subcritical regime, the inception of instabilities in the shear-layer play a major role in the transition to turbulence.

As expected, $\mathrm{KH}$ instabilities frequency increases with the Reynolds number ${ }^{8}$. If the ratio of this frequency to that of the vortex shedding is computed $\left(f_{K H} / f_{v s}\right)$ the obtained dependency with the Reynolds number matches quite well with the scaling obtained by Prasad and Williamson ${ }^{51,52}$ (see figure 14). Earlier studies ${ }^{8,50}$ suggested a ratio proportional $^{2}$ to $R e^{0.5}$, however, Prasad and Williamson ${ }^{51,52}$ proposed a different scaling $\left(f_{K H} / F_{v s} \propto\right.$ $R e^{0.67}$ ) based on the data of experimental measurements by different authors and their own in the range of Reynolds numbers up to $R e<10^{5}$, which later, was confirmed by other researchers (e.g. $f_{K H} / F_{v s} \propto R e^{0.68}$ by Norberg 53 and $f_{K H} / F_{v s} \propto R e^{0.69}$ by Thompson and Hourigan ${ }^{54}$ ). In their analysis, they accounted for the variations of the base pressure and vortex shedding frequency with the Reynolds number, and the upstream motion of 
Forces on a circular cylinder at critical Reynolds

the separation point. As in the present work transition to turbulence occurs just after the separation of the boundary layer, this analysis proves to be valid at least for $R e=$ $2.5 \times 10^{5}-6.5 \times 10^{5}$. This outcome is quite interesting as no direct measurements of these instabilities have been reported so far at $R e>10^{5}$.

\section{CONCLUSIONS}

Large eddy simulations of the flow past a circular cylinder at critical Reynolds numbers of $R e=2.5 \times 10^{5}, 3.8 \times 10^{5}, 5.3 \times 10^{5}, 5.5 \times 10^{5}$ have been performed. To do this, secondorder spectra-consistent numerical schemes on an unstructured grid arrangement have been used. It is well known that in the critical regime transition to turbulence in the separated boundary layer causes the delaying of the separation point and a steep drop in the drag commonly referred to as the drag crisis. This phenomenon here addressed has been well predicted by the present numerical simulations. Measurements of both drag forces and pressure distribution along the cylinder circumference show quite well agreement with the available experimental results. The current simulations also captured the small recirculation bubble formed on the cylinder surface, just after the separated boundary layers transition to turbulence. This laminar separation bubble, which is a fundamental trait of the flow at these critical Reynolds numbers, is first formed on one side of the cylinder surface at $R e=2.5 \times 10^{5}$. With the increase in the Reynolds number, in the present computations at $R e=3.8 \times 10^{5}$, a second bubble starts to form on the other side of the cylinder surface. As a consequence, the flow configuration observed is asymmetric and the symmetry of the flow is recovered as the second bubble settles on the opposite side at $R e=5.3 \times 10^{5}$ and $R e=6.5 \times 10^{5}$. The position, as well as the characteristics dimensions of this flow feature have been measured for all Reynolds numbers.

The changes in the pressure up to a minimum value of the drag coefficient of $C_{D} \approx 0.23$ measured at $R e=6.5 \times 10^{5}$ are also accompanied by changes in the wake topology and in the vortex shedding frequency. It has been shown how the wake width is reduced as the Reynolds number increases and the shear-layers are brought closer with the increase in the pressure gradient. When the shear-layers approach each other as the flow accelerates, they are forced to interact with a higher periodicity thus increasing the vortex shedding frequency up to $S t \approx 0.44$ at $R e=6.5 \times 10^{5}$. This value is in well agreement with the experimental 
Forces on a circular cylinder at critical Reynolds

results at comparable Reynolds numbers; however, whether three-dimensional effects in the wake are the cause of the lost of coherence observed in some experimental measurements still remains an open question.

Regarding the shear-layer frequency, it is found that even at these high Reynolds numbers shear-layer instabilities play a major role in the transition to turbulence. The analysis of the data obtained in the present computations showed that the Reynolds number scaling of the shear-layer frequency is given by $f_{K H} / f_{v s} \propto R e^{0.67}$ which is in agreement with that of Prasad\&Williamson ${ }^{52}$.

\section{ACKNOWLEDGMENTS}

This work has been partially financially supported by the Ministerio de Economía y Competitividad, Secretaría de Estado de Investigación, Desarrollo e Innovación, Spain (ref. ENE2009-07689) and by the Collaboration Project between Universidad Politècnica de Catalunya and Termo Fluids S.L. We acknowledge PRACE (project 2012071290) for awarding us access to the MareNostrum III machine based in Barcelona, Spain.

\section{Appendix A: Sensitiveness of the solutions to the grid resolution}

Numerical simulations are conducted using different levels of refinement. The finer meshes are mainly constructed by refining the wall-normal direction in order to well-solve the boundary layer and capture the near-wall flow features such as the small LSB formed just behind the location of the minimum pressure. Furthermore, in the near-wake zone $-1.0 \leq y / D \leq 1.0$ and $x / D \leq 3$ more control volumes are clustered in order to ensure a mesh as smooth as possible in the region of interest. Details of the different grids used for $R e=5.3 \times 10^{5}$ and the results obtained are summarized in Table V. In the table, together with the mesh size for each level of refinement the value of the largest non-dimensional wall distance $\left(y_{\max }^{+}\right)$is also given.

The drag coefficient, base pressure and Strouhal number obtained with the low-resolution mesh (low-res in Table V) largely deviates from the results obtained with the high-resolution mesh. On the other hand, results are in reasonable agreement for both the medium-resolution and the high-resolution meshes, although the largest differences are observed for the base- 
Forces on a circular cylinder at critical Reynolds

\begin{tabular}{lccccccc}
\hline \hline Mesh & $N C V_{t}\left[\times 10^{6} \mathrm{CVs}\right]$ & $N_{C V}$ plane & $y_{\text {max }}^{+}$ & $-C_{p, \text { min }}$ & $-C_{p, \text { base }}$ & $C_{D}$ & $\mathrm{St}$ \\
\hline \hline high-res & 64 & 500156 & 1.5 & 2.45 & 0.303 & 0.296 & 0.368 \\
medium-res & 48.6 & 379950 & 12 & 2.42 & 0.372 & 0.329 & 0.341 \\
low-res & 38.4 & 299683 & 20 & 1.91 & 0.619 & 0.531 & 0.283 \\
\hline \hline
\end{tabular}

TABLE V: Meshes used for solving the flow at $R e=5.3 \times 10^{5}$ and statistical flow parameters

pressure coefficient. These differences stem from the deviation in the pressure distribution along the cylinder circumference. If the pressure distribution is plotted for all three meshes (see Figure 15), one can notice that the medium-resolution mesh fails in capturing the small recirculation bubble formed on the cylinder surface. This mesh (medium-res in Table V) is not as refined in the wall-normal direction as the high-res mesh, and this is why the LSB cannot be captured by using this grid. Nonetheless, pressure distributions for these grids are in quite good agreement with experimental results from the literature. In the light of these results it is evident that the grid refinement in the vicinity of the cylinder surface is necessary if the LSB is to be captured. Thus, the meshes used in the numerical simulations presented throughout this paper were especially refined in the near-wall direction depending on the Reynolds number to be solved.

\section{REFERENCES}

${ }^{1}$ J. H. Gerrard, "The mechanics of the formation region of vortices behind bluff bodies," J. Fluid Mech. 25, 401-413 (1966).

${ }^{2}$ A. Roshko, "Experiments on the flow past a circular cylinder at very high Reynolds number," J. Fluid Mech. 10, 345-356 ( 1961).

${ }^{3}$ C. Wieselsberger, "New data on the laws of fluid resistance," Tech. Rep. NACA TN-84 NACA, 1922.

${ }^{4}$ A. Fage, "Drag of circular cylinders and spheres," Tech. Rep. R\&M 1370 Aeronautical Reasearch Committee, 1930.

${ }^{5}$ E. Achenbach and E. Heinecke, "On vortex shedding from smooth and rough cylinders in 
Forces on a circular cylinder at critical Reynolds

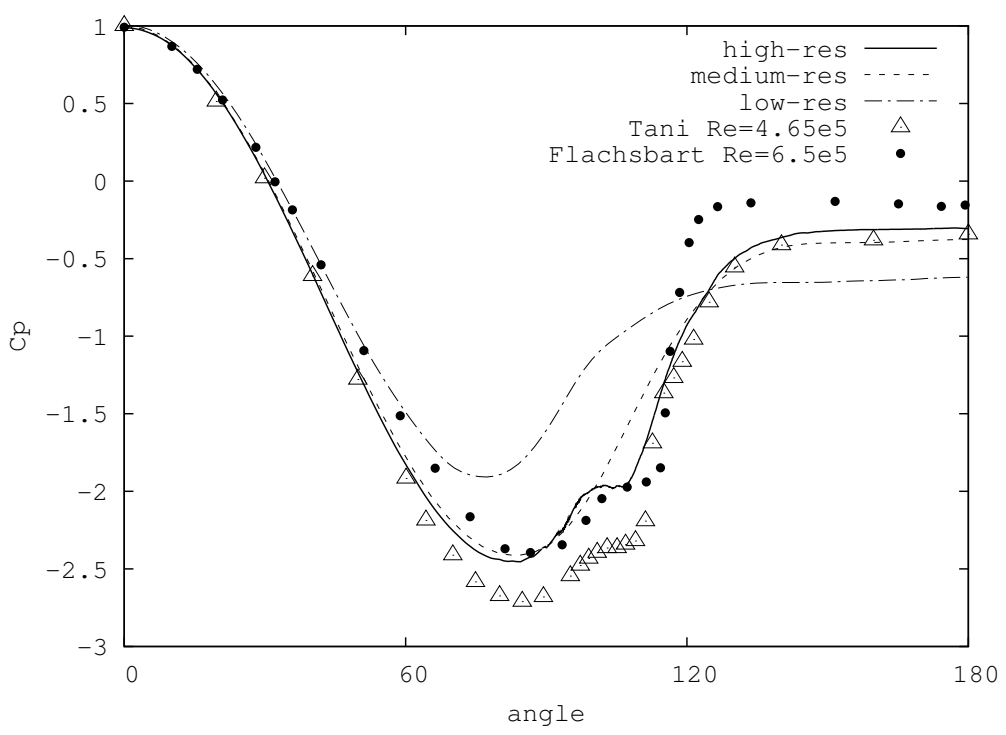

FIG. 15: Comparison of the pressure coefficient on the cylinder surface for the different meshes under study at $R e=5.3 \times 10^{5}$

the range of Reynolds numbers 6e3 to 5e6," J. Fluid Mech. 109, 239-251 (1981).

${ }^{6}$ C. H. K. Williamson, "Vortex Dynamics in the Cylinder Wake," Annu. Rev. Fluid Mech. 28, 477-539 (1996).

${ }^{7}$ C. Norberg, "Fluctuating lift on a circular cylinder: review and new measurements," J. Fluids Struct. 17, 57-96 (2003).

${ }^{8}$ M.S. Bloor, "The transition to turbulence in the wake of a circular cylinder," J. Fluid Mech. 19, 290-304 (1964).

${ }^{9}$ W.J. Bursnall and L.K. Jr Loftin, "Experimental investigation of the pressure distribution about a yawed circular cylinder in the critical Reynolds number range," Tech. Rep. TN2463, NACA, 1951.

${ }^{10}$ P.W. Bearman, "On vortex shedding from a circular cylinder in the critical Reynolds number regime," J. Fluid Mech. 37, 577-585 (1969).

${ }^{11} \mathrm{G}$. Schewe, "On the force fluctuations acting on a circular cylinder in crossflow from subcritical up to transcritical Reynolds numbers," J. Fluid Mech. 133, 265-285 (1983).

${ }^{12}$ I. Tani, "Low-speed flows involving bubble separations," Prog. Aerosp. Sci., 70-103(1964). ${ }^{13}$ M. Alam and N.D. Sandham, "Direct numerical simulation of short separation bubbles with turbulent reattachment," J. Fluid Mech. 410, 1-28 (2000). 
Forces on a circular cylinder at critical Reynolds

${ }^{14} \mathrm{E}$. Achenbach, "Distribution of local pressure and skin friction around a circular cylinder in cross-flow up to Re=5e6," J. Fluid Mech. 34, 625-639 (1968).

${ }^{15}$ N.K. Delany and N.E. Sorensen, "Low-speed drag of cylinders of various shapes," Tech. Rep. TN3038, NACA, 1953.

${ }^{16}$ W.C.L. Shih, C. Wang, D. Coles, and A. Roshko, "Experiments on flow past rough circular cylinders at large Reynolds numbers," J Wind Eng. Ind. Aerod. 49, 351-368 (1993).

${ }^{17}$ J.W.G. Van Nunen, "Pressure and forces on a circular cylinder in a cross flow at high Reynolds numbers," in Flow Induced Structural Vibrations, edited by Springer-Verlag (Berlin, 1974) pp. 748-754.

${ }^{18}$ G.W. Jones, "Unsteady lift forces generated by vortex shedding about a large, stationary, and oscillating cylinder at high Reynolds numbers," Tech. Rep. NASA-TM-X-61214 NASA Langley Research Center, 1968.

${ }^{19}$ A. Fage and V.M. Falkner, "Further experiments on the flow around a circular cylinder," Tech. Rep. R\&M 1369 Aeronautical Reasearch Committee, 1931.

${ }^{20}$ X. Ma, G.S. Karamanos, and G.E. Karniadakis, "Dynamics and low-dimensionality of a turbulent wake," J. Fluid Mech. 410, 29-65 (2000).

${ }^{21}$ S. Dong, G.E. Karniadakis, A. Ekmekci, and D. Rockwell, "A combined direct numerical simulation-particle image velocimetry study of the turbulent near wake," J. Fluid Mech. 569, 185-207 (2006).

${ }^{22}$ O. Lehmkuhl, I. Rodríguez, R. Borrell, and A. Oliva, "Low-frequency unsteadiness in the vortex formation region of a circular cylinder," Phys. Fluids 25, 085109 (2013).

${ }^{23}$ I. Celik and F.D. Shaffer, "Long time-averaged solutions of turbulent flow past a circular cylinder," J. Wind Eng. Ind. Aerod. 56, 185-212 (1995).

${ }^{24}$ G. Vaz, C. Mabilat, R. van der Wal, and P. Gallagher, "Viscous flow computations on a smooth cylinders: A detailed numerical study with validation," in ASME 2007 26th International Conference on Offshore Mechanics and Arctic Engineering, edited by ASME Digital Collection (San Diego, California, 2007) pp. 849-860.

${ }^{25}$ A. Travin, M. Shur, M. Strelets, and P. Spalart, "Detached-eddy simulations past a circular cylinder," Flow Turbul. Combust. 63, 293-313 (2000).

${ }^{26}$ C. Fureby, "ILES and LES of Complex Engineering Turbulent Flows," J.Fluid Eng. 129, 1514-1523 (2007).

${ }^{27}$ M. Minguez, R. Pasquetti, and E. Serre, "High-order large-eddy simulation of flow over 
Forces on a circular cylinder at critical Reynolds

the Ahmed body car model," Phys. Fluids 20, 095101 (2008).

${ }^{28}$ O. Lehmkuhl, I. Rodríguez, A. Baez, A. Oliva, and C.D. Pérez-Segarra, "On the large-eddy simulations for the flow around aerodynamic profiles using unstructured grids," Comput. Fluids 84, 176-189 (2013).

${ }^{29}$ M. Breuer, "A challenging test case for large eddy simulation: high Reynolds number circular cylinder flow," Int. J. Heat Fluid Flow 21, 648-654 (2000).

${ }^{30} \mathrm{P}$. Catalano, M. Wang, G. Iaccarino, and P. Moin, "Numerical simulation of the flow around a circular cylinder at high Reynolds numbers," Int. J. Heat Fluid Flow 24, 463469 (2003).

${ }^{31} \mathrm{~S}$. Singh and S. Mittal, "Simulation of drag crisis in flow past a circular cylinder using 2D computations," J. of Aerosp. Sci. Tech. 55, 56-62 (2003).

${ }^{32}$ S.J. Karabelas, "Large Eddy Simulation of high-Reynolds number flow past a rotating cylinder," Int. J. Heat Fluid Flow 31, 518-527 (2010).

${ }^{33}$ C. Moussaed, M.V. Salvetti, S. Wornom, B. Koobus, and A. Dervieux, "Simulation of the flow past a circular cylinder in the supercritical regime by blending RANS and variationalmultiscale LES models," J. Fluid. Struct., 47, 114-123(2014).

${ }^{34}$ F. Nicoud and F. Ducros, "Subgrid-scale stress modelling based on the square of the velocity gradient tensor," Flow Turbul. Combust. 62, 183-200 (1999).

${ }^{35}$ I. Rodríguez, R. Borrell, O. Lehmkuhl, C.D. Pérez-Segarra, and A. Oliva, "Direct Numerical Simulation of the Flow Over a Sphere at Re = 3700," J. Fluid Mech. 679, 263-287 (2011).

${ }^{36}$ I. Rodríguez, O. Lehmkuhl, R. Borrell, and A. Oliva, "Flow dynamics in the wake of a sphere at sub-critical Reynolds numbers," Comput. Fluids 80, 233-243 (2013).

${ }^{37}$ D.E. Aljure, O. Lehmkhul, I. Rodríguez, and A. Oliva, "Flow and Turbulent Structures Around Simplified Car Models," Comput. Fluids 96, 122-135 (2014).

${ }^{38}$ R.W.C.P. Verstappen and A.E.P. Veldman, "Symmetry-preserving discretization of turbulent flow," J. Comput. Phys. 187, 343-368 (2003).

${ }^{39}$ F.X. Trias and O. Lehmkuhl, "A self-adaptive strategy for the time integration of NavierStokes equations," Numer. Heat Transfer, Part B 60, 116-134 (2011).

${ }^{40}$ L. Jofre, O. Lehmkuhl, J. Ventosa, F.X. Trias, and A. Oliva, "Conservation properties of unstructured finite-volume mesh schemes for the Navier-Stokes equations," Numer. Heat Transfer, Part B: Fundamentals 54, 53-79 (2014). 
Forces on a circular cylinder at critical Reynolds

${ }^{41}$ F.X. Trias, O. Lehmkuhl, A. Oliva, C.D. Pérez-Segarra, and R.W.C.P. Verstappen, "Symmetry-preserving discretization of NavierStokes equations on collocated unstructured grids," J. Comput. Phys. 258, 246-267 (2014).

${ }^{42}$ R. Borrell, O. Lehmkuhl, F.X. Trias, and A. Oliva, "Parallel direct Poisson solver for discretisations with one Fourier diagonalisable direction," J. Comput. Phys. 230, 47234741 (2011).

${ }^{43}$ R.E. Spitzer, Measurements of unsteady pressures and wake fluctuations for flow over a cylinder at supercritical Reynolds number, Ph.D. thesis, California Institute of Technology (1964).

${ }^{44}$ B. Cantwell and D. Coles, "An experimental study of entrainment and transport in the turbulent near wake of a circular cylinder," J. Fluid Mech. 136, 321-374 (1983).

${ }^{45}$ J.C.R. Hunt, A.A. Wray, and P. Moin, "Eddies, stream and convergence zones in turbulent flows," Tech. Rep. CTR-S88 (Center for turbulent research, 1988).

${ }^{46}$ P. Parnaudeau, J. Carlier, D. Heithz, and E. Lamballais, "Experimental and numerical studies of the flow over a circular cylinder at Reynolds number 3900," Phys. Fluids 20, 085101 (2008).

${ }^{47}$ N.R. Lomb, "Least-squares frequency analysis of unequally spaced data.." Astrophys. Space Sci. 39, 447-462 (1976).

${ }^{48}$ M.F. Unal and D. Rockwell, "The role of shear layer stability in vortex shedding from cylinders," Phys. Fluids 27, 2598 (1984).

${ }^{49}$ T. Wei and C.R. Smith, "Secondary vortices in the wake of circular cylinders," J. Fluid Mech. 169, 513-533 (1986).

${ }^{50}$ A. Kourta, H.C. Boisson, P. Chassaing, and H.H. Minh, "Nonlinear interaction and the transition to turbulence in the wake of a circular cylinder," J. Fluid Mech. 181, 141-161 (1987).

${ }^{51}$ A. Prasad and C.H.K. Williamson, "The instability of the separated shear layer from a bluff body," Phys. Fluids 8, 1347 (1996).

${ }^{52}$ A. Prasad and C.H.K. Williamson, "The instability of the shear layer separating from a bluff body," J. Fluid Mech., 375-492(1997).

${ }^{53} \mathrm{C}$. Norberg, "LDV measurements in the near wake of a circular cylinder," in Advances in Understanding of Bluff Body Wakes and Flow-Induced Vibrations, edited by ASME Fluids Engineering Division, (Washington DC, 1998) paper 42. 
Forces on a circular cylinder at critical Reynolds

${ }^{54}$ M.C. Thompson and K. Hourigan, "The shear-layer instability of a circular cylinder wake," Phys. Fluids 17, 021702 (2005). 